\title{
Star formation around RCW 120, the perfect bubble ${ }^{\star}$
}

\author{
L. Deharveng ${ }^{1}$, A. Zavagno ${ }^{1}$, F. Schuller ${ }^{2}$, J. Caplan ${ }^{1}$, M. Pomarès ${ }^{1}$, and C. De Breuck ${ }^{3}$ \\ ${ }^{1}$ Laboratoire d'Astrophysique de Marseille (UMR 6110 CNRS \& Université de Provence), 38 rue F. Joliot-Curie, \\ 13388 Marseille Cedex 13, France \\ e-mail: Lise.Deharveng@oamp.fr \\ 2 Max-Planck-Institut für Radioastronomie, Auf dem Hügel 69, 53121 Bonn, Germany \\ 3 European Southern Observatory, Karl-Schwarschild Strasse, 85748 Garching bei München, Germany
}

Received 12 November 2008 / Accepted 7 January 2009

\begin{abstract}
Context. This study deals with the star formation triggered by H II regions.

Aims. We wish to take advantage of the very simple morphology of RCW 120 - a perfect bubble - to understand the mechanisms triggering star formation around an $\mathrm{H}$ II region and to establish what kind of stars are formed there.

Methods. We present $870 \mu \mathrm{m}$ observations of RCW 120, obtained with the APEX-LABOCA camera. These show the distribution of cold dust, and thus of neutral material. We use Spitzer-MIPS observations at $24 \mu \mathrm{m}$ and $70 \mu \mathrm{m}$ to detect the young stellar objects present in this region and to estimate their evolutionary stages.

Results. A layer of dense neutral material surrounds the entire H II region, having been swept up during the region's expansion. This layer has a mass greater than $2000 M_{\odot}$ and is fragmented, with massive fragments elongated along the ionization front (IF). We measured the $24 \mu \mathrm{m}$ flux of 138 sources. Of these, 39 are Class I or flat-spectrum young stellar objects (YSOs) observed in the direction of the collected layer. We show that several triggering mechanisms are acting simultaneously in the swept-up shell, where they form a second generation of stars. No massive YSOs are detected. However, a massive, compact $870 \mu \mathrm{m}$ core lies adjacent to the IF. A $70 \mu \mathrm{m}$ source with no $24 \mu \mathrm{m}$ counterpart is detected at the same position. This source is a likely candidate for a Class 0 YSO. Also at $24 \mu \mathrm{m}$, we detect a chain of about ten regularly spaced Class I or flat spectrum sources, parallel to the IF, in the direction of the most massive fragment. We suggest that the formation of these YSOs is the result of Jeans gravitational instabilities in the collected layer. Finally, the $870 \mu \mathrm{m}$ emission, the $24 \mu \mathrm{m}$ emission, and the $\mathrm{H} \alpha$ emission show the existence of an extended and partially ionized photodissociation region around RCW 120. This demonstrates the long-distance influence of the H II region upon its surrounding medium.
\end{abstract}

Key words. stars: formation - stars: early-type - ISM: H II regions - ISM: individual objects: RCW 120

\section{Introduction}

RCW 120 is perhaps the most perfect of the many bubbles detected in the Galactic plane by Spitzer at $8.0 \mu \mathrm{m}$ (Churchwell 2006, bubble S7). This H II region presents a simple morphology, with a well defined ionization front (IF) separating the ionized and the surrounding neutral material. This optical H II region has the additional advantage of lying nearby, at a distance of $1.34 \mathrm{kpc}$, and being isolated. Its distance is reliable as the photometric and kinematic determinations are in good agreement (Zavagno et al. 2007, hereafter ZA07). The J2000 coordinates of its O8 central exciting star are $l=348^{\circ} .2239, b=+0^{\circ} .4643$, $\alpha=17^{\mathrm{h}} 12^{\mathrm{m}} 20^{\mathrm{s}} 6, \delta=-38^{\circ} 29^{\prime} 26^{\prime \prime}$. This star is identified in ZA07's Fig. 1.

Star formation associated with RCW 120 has been studied by ZA07, mainly using observations obtained at $1.2 \mathrm{~mm}$, supplemented by 2MASS and Spitzer-GLIMPSE data. ZA07 have shown the presence of a layer of collected neutral material surrounding the ionized gas. This layer is fragmented, with massive fragments adjacent to the ionized gas, and elongated along the

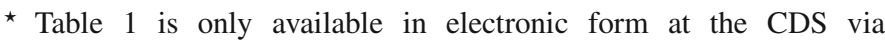
anonymous ftp to cdsarc.u-strasbg.fr (130.79.128.5) or via http://cdsweb.u-strasbg.fr/cgi-bin/qcat?J/A+A/496/177
IF. Many young stellar objects (YSOs) are present in the vicinity of the H II region. However no massive object is observed, even in the direction of the most massive fragment. ZA07's conclusion is that the collect \& collapse process is taking place around RCW 120, but that this region is too young for massive-star formation to take place via gravitational instabilities along the collected layer.

Since then, new observations have become available, allowing us to progress in our understanding of star formation triggered by RCW 120. These observations are: $i$ ) a deep map of the dust emission at $870 \mu \mathrm{m}$, obtained with the APEX-LABOCA camera during the science verification phase, giving improved knowledge of the cold dust distribution and hence of the neutral material associated with RCW 120; ii) Spitzer-MIPSGAL frames at $24 \mu \mathrm{m}$ and $70 \mu \mathrm{m}$, allowing a better detection of the YSOs possibly associated with RCW 120, and a better determination of their evolutionary stages. The $870 \mu \mathrm{m}$ observation is presented in Sect. 2.1, and the $24 \mu \mathrm{m}$ and $70 \mu \mathrm{m}$ frames and their photometric reduction in Sect. 2.2. Section 3 deals with the distribution of the neutral material around RCW120. The nature of the YSOs associated with RCW 120 is discussed in Sect. 4. In Sect. 5 we try to understand the interactions between the central 
$\mathrm{H}$ II region and the surrounding material, and how these interactions influence the star formation in this region.

Additional details about RCW 120, and various composite colour images, can be found in Deharveng \& Zavagno (2008).

\section{New observations}

\subsection{APEX-LABOCA observations at $870 \mu \mathrm{m}$}

The APEX ${ }^{1}$-LABOCA camera was used at $870 \mu \mathrm{m}(345 \mathrm{GHz})$ to observe the continuum emission of the cold dust associated with RCW 120. The Large Apex BOlometer CAmera, commissioned in May 2007, is a 295-pixel bolometer array developed by the Max-Planck-Institut für Radioastronomie (Siringo et al. 2007). The APEX beam size at $870 \mu \mathrm{m}$ is 19.2 .

RCW 120 was observed in July 2007 as part of the science verification program. A $15^{\prime} \times 15^{\prime}$ map was obtained in the spiral raster mode. The on-source observing time was less than two hours. During these observations the amount of precipitable water vapour was $\sim 1 \mathrm{~mm}$.

The observations were reduced using the Bolometer array data Analysis package (BoA; Schuller et al. in preparation). The reduction steps involved in the processing of the data are: flux calibration and opacity correction, deduced from skydip measurements and observations of primary and secondary calibrators; flagging of bad bolometers; correlated noise removal; despiking; low-frequency filtering. As a result of the correction for correlated noise, uniformly extended emission (on scales larger than 2.5), which mimics the variations of the sky emission (skynoise), is filtered out.

The calibration uncertainty in the final map is of the order of $15 \%$. The pointing uncertainty is $\sim 4^{\prime \prime}(0.025 \mathrm{pc}$ at the distance of RCW 120). We estimate the rms noise of the final image to be $0.02 \mathrm{Jy} /$ beam.

\subsection{Spitzer-MIPS observations at $24 \mu \mathrm{m}$ and $70 \mu \mathrm{m}$}

Figure 1 is a composite colour image of RCW 120, from the optical to the mid-IR. Blue is the $\mathrm{H} \alpha$ emission of the ionized gas, from SuperCOSMOS (Parker et al. 2005). Green is the $8.0 \mu \mathrm{m}$ emission from Spitzer-GLIMPSE (Benjamin et al. 2003). At this wavelength we mainly see the emission of polycyclic aromatic hydrocarbons (PAHs). These big molecules are destroyed inside the HII region by the UV radiation of the exciting stars. They are excited in the photodissociation region (PDR) by the radiation leaking from the HII region. Thus they are good tracers of ionization fronts. Red shows the emission at $24 \mu \mathrm{m}$, from Spitzer-MIPSGAL (Carey et al. 2005). We see both the extended emission of the small dust grains present inside the ionized region and the PDR, and the emission of grains present in the envelopes of YSOs and evolved stars, hence the usefulness of the $24 \mu \mathrm{m}$ images for detecting YSOs. (The central $24 \mu \mathrm{m}$ extended emission is saturated.)

We have measured three Post BCD frames from the SpitzerMIPSGAL survey at $24 \mu \mathrm{m}$. These cover a zone going from $257^{\circ} .85$ to $258^{\circ} .35$ in right ascension and from $-38^{\circ} .70$ to $-38^{\circ} .20$ in declination, encompassing the zone of influence of RCW 120. We used the DAOPHOT stellar photometry package with PSF fitting (Stetson 1987). The main difficulty to overcome is the

\footnotetext{
1 This publication is based on data acquired with the Atacama Pathfinder EXperiment (APEX), in run 078.F-9005(A). APEX is a collaboration between the Max-Planck-Institut für Radioastronomie, the European Southern Observatory, and the Onsala Space Observatory.
}

measurement of faint stars superimposed on a bright and very irregular background. For this we used an iterative process. A first run of DAOPHOT, using the normal procedures, yields stellar positions and rough magnitudes. These stars are then subtracted from the original frame. The resulting image is smoothed with a median filter (window of $11 \times 11$ pixels, larger than the size of a star) to remove any vestiges of the stars, and obtain an image of the background emission alone. This background image is then subtracted from the original frame. On the resulting image the stars lie on a low brightness and more uniform background (the only remaining structures are those smaller than the filter window). Further reduction is performed by the normal DAOPHOT procedures. This iterative process can be repeated several times until convergence is satisfactory. We check the result by eye; if the reduction is good no brightness peaks or holes should be seen at the positions of the stars after these stars have been subtracted from the original frame.

The $24 \mu \mathrm{m}$ frames overlap, providing us with double values of the magnitudes of 27 sources. This allows us to estimate the accuracy of the results: the rms magnitude difference for these 27 pairs is $0.11 \mathrm{mag}$. The DAOPHOT PSF magnitudes are then converted into aperture magnitudes, following the "Quick Point Source Photometric Measurement" procedure (http://ssc. spitzer. caltech.edu/archanaly/quickphot.html), and using isolated bright stars. The resulting $24 \mu \mathrm{m}$ magnitudes, obtained for 138 sources, are given in Table 1.

We have also reduced three $70 \mu \mathrm{m}$ Post BCD frames, from the Spitzer-MIPSGAL survey. Six sources have been measured manually, using aperture photometry. These measurements are also given in Table 1.

We have used the interactive software sky atlas Aladin ${ }^{2}$ to superimpose the 2MASS catalogue on the GLIMPSE and $24 \mu \mathrm{m}$ images. In the few cases where several GLIMPSE or 2MASS sources were observed in the direction of a $24 \mu \mathrm{m}$ source, only the central one was considered.

Table 1, which is available in electronic form at the CDS, presents our measured $24 \mu \mathrm{m}$ magnitudes along with these objects' $1.25 \mu \mathrm{m}$ to $8.0 \mu \mathrm{m}$ magnitudes taken from the 2MASS and Spitzer-GLIMPSE catalogues. Column 1 gives an identification number. Columns 2 and 3 are the coordinates according to the Spitzer-GLIMPSE catalogues. Columns 4 to 10 give the $J, H, K,[3.6],[4.5]$, [5.8], and [8.0] magnitudes. These are extracted from the (highly reliable) GLIMPSE I Spring '07 Catalog. When necessary they have been supplemented with values from the (less reliable) GLIMPSE I Spring '07 Archive, and by the 2MASS All-Sky Point Source Catalog (a colon indicates an uncertain measurement). Columns 11 and 12 give our $24 \mu \mathrm{m}$ and $70 \mu \mathrm{m}$ measurements. Five bright sources which have [24] $\leq 1.38$ mag (as indicated by a colon) are clearly saturated at $24 \mu \mathrm{m}$. Column 13 gives our conclusions concerning the evolutionary stages of the sources (see Sect. 4).

\section{The distribution of neutral material around RCW 120}

The distribution of the 1.2-mm dust emission was presented and discussed by ZA07 using SEST-SIMBA data (beam size 24", rms noise $\sim 0.02 \mathrm{Jy} /$ beam, exposure time $10 \mathrm{~h}$ ). Here we present, in Fig. 2, the distribution of the $870 \mu \mathrm{m}$ dust emission, obtained with the APEX-LABOCA camera (beam size 19!'2, rms noise $\sim 0.02 \mathrm{Jy} /$ beam, exposure time $<2 \mathrm{~h}$ ).

\footnotetext{
2 http://aladin.u-strasbg.fr/
} 


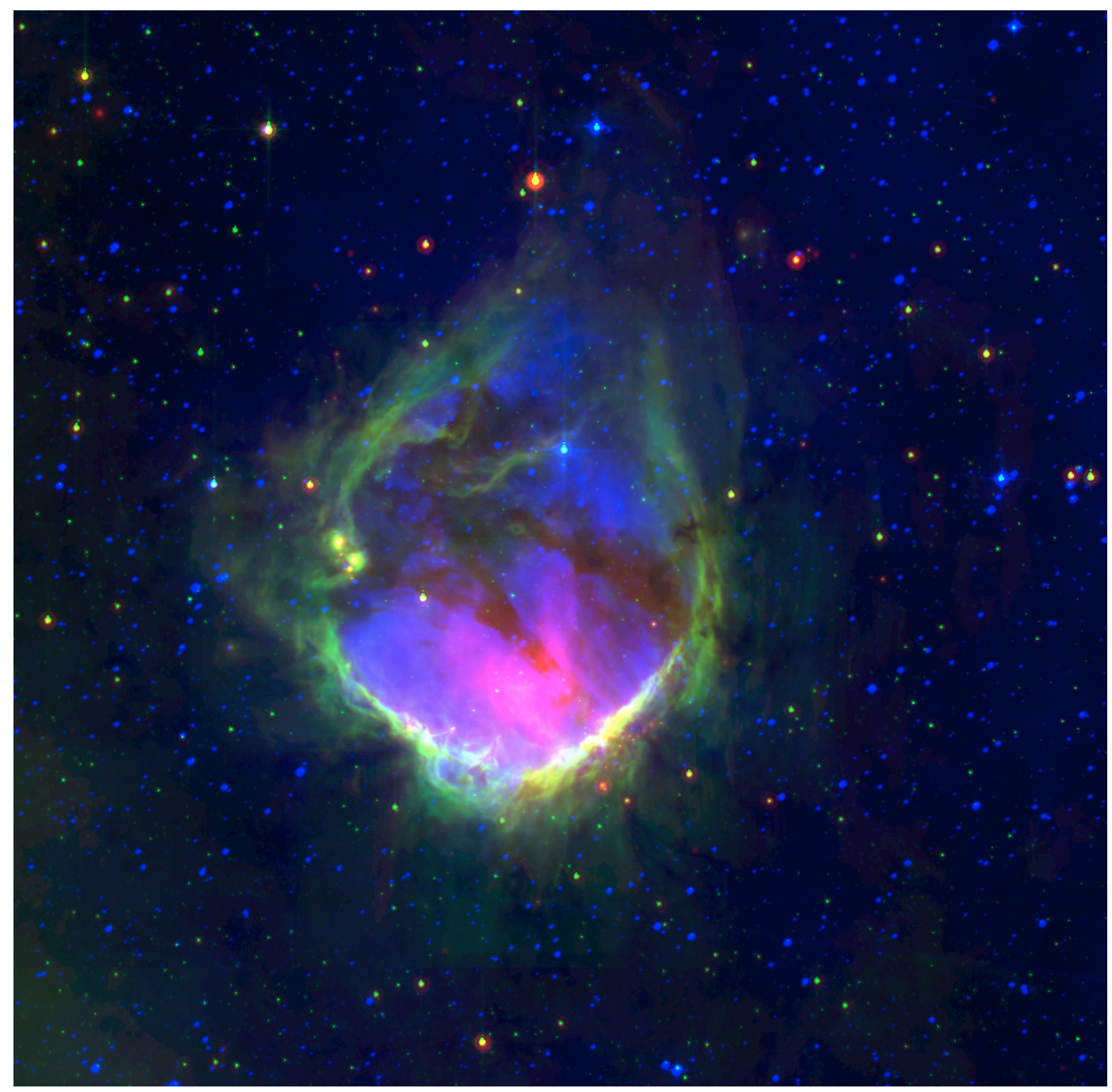

Fig. 1. Colour composite image of RCW 120. Blue is $\mathrm{H} \alpha$ emission, green is the PAH emission at $8.0 \mu \mathrm{m}$ and red is the $24 \mu \mathrm{m}$ emission of small dust grains (see text). North is up and east is left. The field size is $\sim 24^{\prime}(\mathrm{N}-\mathrm{S}) \times 25^{\prime}(\mathrm{E}-\mathrm{W})$.

Emission at $870 \mu \mathrm{m}$ is mainly the thermal continuum of cold dust. Contamination from the ${ }^{12} \mathrm{CO}(3-2)$ line may occur, but this emission is expected to be faint (see the discussion in Schuller et al. in preparation).

The $870 \mu \mathrm{m}$ map clearly resembles the 1.2-mm map: a layer of cold dust surrounds the ionized region; this layer is fragmented, with condensations elongated along the ionization front, especially in the south. The higher resolution and higher signalto-noise ratio at $870 \mu \mathrm{m}$ reveal new structures that were only barely visible in the 1.2-mm map. This is the case for the radial structures that are observed north-east of the ionized region, and for the filaments responsible for the absorption in the central parts of RCW 120 (the central absorbing filaments are conspicuous on Fig. 2, right, blue channel). Also, small faint condensations are now revealed, these often being associated with Class I sources detected thanks to the GLIMPSE and MIPSGAL surveys (Sect. 4).

\subsection{Mass determination}

Assuming that the $870 \mu \mathrm{m}$ emission is uniquely thermal dust emission, we can derive the mass of the emitting material. According to Hildebrand (1983) the total (gas+dust) mass of a condensation is related to its flux density $S_{v}$ by

$$
M_{\text {(gas+dust) }}=100 \frac{S_{870 \mu \mathrm{m}} D^{2}}{\kappa_{870 \mu \mathrm{m}} B_{870 \mu \mathrm{m}}\left(T_{\text {dust }}\right)}
$$

where $D$ is the distance of the source, $\kappa_{870 \mu \mathrm{m}}$ is the dust opacity per unit mass at $870 \mu \mathrm{m}$, and $B_{870 \mu \mathrm{m}}\left(T_{\text {dust }}\right)$ is the Planck function for a temperature $T_{\text {dust }}$. We have assumed a gas-to-dust ratio of 100 . The dust temperature is taken as $20 \mathrm{~K}$ or $30 \mathrm{~K}$, as often assumed for protostellar condensations (cf. Motte et al. 2003, Johnstone et al. 2006). Following Ossenkopf \& Henning (1994) we adopt the value $\kappa_{870 \mu \mathrm{m}}=1.8 \mathrm{~cm}^{2} \mathrm{~g}^{-1}$. The dust opacity depends strongly on the dust grains' coating (ice mantles), compactness, coagulation evolution and the ambient density (see their Fig. 5a-c). This value agrees reasonably well with those adopted by various authors to derive masses from SCUBA $850 \mu \mathrm{m}$ emission maps (e.g. Kirk et al. 2006; Young et al. 2003).

Table 2 lists the measured and derived properties obtained for several structures discussed in the text (Sect. 5). Column 1 gives the structure's name. Condensations \#1 to \#8 are those identified in ZA07 (their Fig. 4). Columns 2 and 3 give the emission peak coordinates and Col. 4 gives the emission peak level. Columns 5 and 6 give the intensity value used to define the integration boundary and hence the flux density. The choice of 

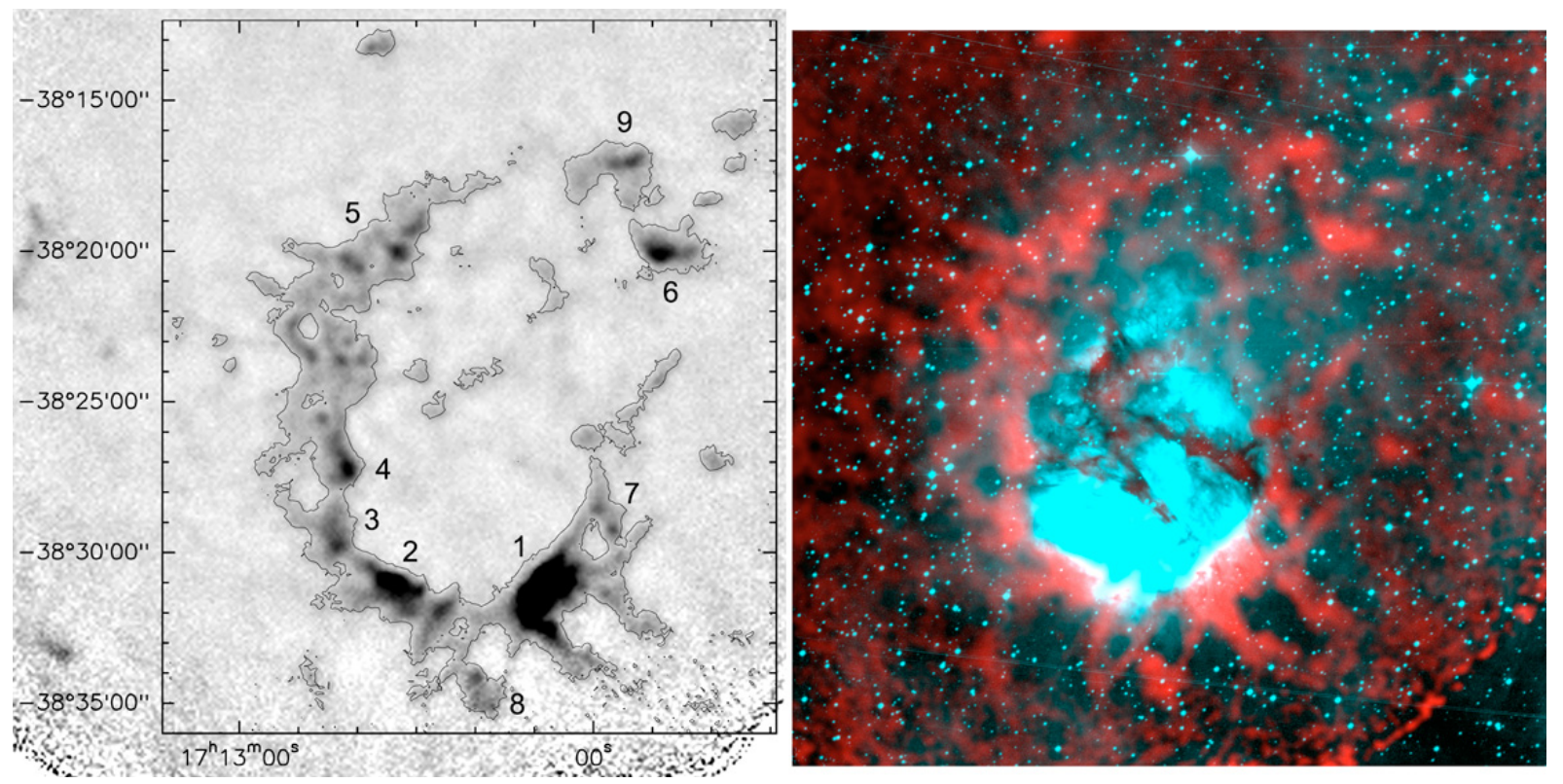

Fig. 2. Left: $870 \mu \mathrm{m}$ emission, observed with APEX-LABOCA. The contour corresponds to the $5 \sigma$ level of $0.1 \mathrm{Jy} /$ beam. The dust condensations are identified by a number used in ZA07 and in Table 2. Right: colour composite image of RCW 120: the $870 \mu \mathrm{m}$ emission appears in red, the $\mathrm{H} \alpha$ emission in turquoise.

the levels used to delineate the structures and measure their flux densities is somewhat arbitrary; they have been chosen in order to separate the different structures. Finally Col. 7 gives the derived masses depending on the adopted temperature, 20 or $30 \mathrm{~K}$; the lower mass corresponds to the higher dust temperature. The masses are highly uncertain. As shown by Col. 7, the dust temperature uncertainty alone results in a mass uncertainty of a factor two. But the dust opacity also is uncertain by a factor of two (Henning et al. 2005), which implies an uncertainty of the same order for the mass.

The masses we estimate from the $870 \mu \mathrm{m}$ emission map are roughly twice those obtained by ZA07 from the 1.2-mm map. This discrepancy is partly due to the values adopted for the dust opacity, and mostly to the levels used to delineate the condensations. The new values are more reliable, as the $870 \mu \mathrm{m}$ map has a much better signal-to-noise ratio; also, the structures for which we estimate the masses are better defined in this paper.

In order to derive the mass contained in the annular structure that surrounds the ionized region (referred to as "Shell" in Table 2) we integrated the flux over the surface enclosed by the $5 \sigma$ level contour (corresponding to $0.1 \mathrm{Jy} /$ beam; see Fig. 2). The result is a total mass for the shell in the range 1200-2100 $M_{\odot}$.

Condensation 1 contains a very bright core (emission peak of $13.3 \mathrm{Jy} /$ beam) superimposed on a bright elongated plateau at a level $\sim 2 \mathrm{Jy} /$ beam. This core is resolved, with a deconvolved size of 14 .' $6 \times 7$ 7.'7 (0.095 pc $\times 0.05$ pc; FWHM; see Sect. 5.3, condensation 1 ).

In addition to the condensations listed in ZA07, a few other structures were measured, most of them associated with Class I sources. They are identified in Figs. 10 to 15.

\subsection{Column density and extinction}

We have calculated the $\mathrm{H}_{2}$ column density, $N\left(\mathrm{H}_{2}\right)$, from the surface brightness $F_{870 \mu \mathrm{m}}$, using the formula

$$
N\left(\mathrm{H}_{2}\right)=\frac{100 F_{870 \mu \mathrm{m}}}{\kappa_{870 \mu \mathrm{m}} B_{870 \mu \mathrm{m}}\left(T_{\text {dust }}\right) 2.3 m_{\mathrm{H}} \Omega_{\text {beam }}},
$$

where $F_{870 \mu \mathrm{m}}$ is expressed in Jy beam ${ }^{-1}, B_{870 \mu \mathrm{m}}$ in Jy, $N\left(\mathrm{H}_{2}\right)$ is per square centimetre, the hydrogen atom mass $m_{\mathrm{H}}$ is in grams and the beam solid angle $\Omega_{\text {beam }}$ is in steradians.

Adopting $\Omega_{\text {beam }}=9.817 \times 10^{-9} \mathrm{sr}$ corresponding to a beam of 19.2 (FWHM), and assuming $T_{\text {dust }}=20 \mathrm{~K}$, this gives

$N\left(\mathrm{H}_{2}\right)=3.136 \times 10^{22} F_{870 \mu \mathrm{m}}$.

From the classical relations $N\left(\mathrm{H}+\mathrm{H}_{2}\right) / E(B-V)=5.8 \times$ $10^{21}$ particles $\mathrm{cm}^{-2} \mathrm{mag}^{-1}$ (Bohlin et al. 1978) and $A_{V}=$ $3.1 E(B-V)$, we obtain $A_{V}=5.34 \times 10^{-22} N\left(\mathrm{H}_{2}\right)$.

The bright core in condensation 1 , with its emission peak of $13.3 \mathrm{Jy} /$ beam, is responsible for a visual extinction of $225 \mathrm{mag}$ (assuming a dust temperature of $20 \mathrm{~K}$ ). Similarly, all the material enclosed by the $5 \sigma$ contour (Fig. 2, $0.1 \mathrm{Jy} /$ beam level) corresponds to a visual extinction greater than $1.7 \mathrm{mag}$. Dust filaments - very conspicuous in front of the ionized region are responsible for the absorption observed at optical wavelengths in the direction of the centre of RCW 120 (e.g. at $\mathrm{H} \alpha$, in Fig. 2). The material observed at $870 \mu \mathrm{m}$ in the direction of the exciting star of the H II region has a small column density, corresponding to a visual extinction $\leq 1 \mathrm{mag}$. This is less than the extinction, $A_{V}=4.65 \mathrm{mag}$, determined by Avedisova $\&$ Kondratenko (1984) for the exciting star. The relatively large beam of APEX-LABOCA smoothes out small-scale local features of the dust emission. Also, large-scale structures are removed during the reduction (Sect. 2). The visual extinction is probably larger than estimated using APEX data, by some 3-4 mag.

The Spitzer emission maps at $8.0 \mu \mathrm{m}$ and at $24 \mu \mathrm{m}$ show, in absorption, several infrared dark clouds (IRDCs) in the vicinity of RCW 120. When we compare these maps with the dust emission map at $870 \mu \mathrm{m}$, Fig. 3, we see that most of the cold dust condensations in the north have IRDCs as counterparts, whereas in the south the brighter cold dust condensations have no IRDC counterparts. This gives an indication of the respective positions of the dust condensations and the ionized gas: in the north, the dust condensations are in front of the PDR, whereas they are slightly behind the ionization front in the south. 
Table 2. Mass estimates.

\begin{tabular}{|c|c|c|c|c|c|c|}
\hline \multirow[t]{2}{*}{ Number } & \multicolumn{2}{|c|}{ Peak position } & \multirow{2}{*}{$\begin{array}{c}F_{870 \mu \mathrm{m}}^{\text {peak }} \\
(\mathrm{Jy} / \text { beam })\end{array}$} & \multirow{2}{*}{$\begin{array}{c}\text { Boundary level } \\
\text { (Jy/beam) }\end{array}$} & \multirow{2}{*}{$\begin{array}{c}F_{870 \mu \mathrm{m}}^{\mathrm{int}} \\
(\mathrm{Jy})\end{array}$} & \multirow{2}{*}{$\begin{array}{c}\text { Mass range }^{1} \\
\left(M_{\odot}\right)\end{array}$} \\
\hline & $\alpha_{2000}$ & $\delta_{2000}$ & & & & \\
\hline Shell & & & & 0.1 & 190 & $1920-1100$ \\
\hline Condensation 1 & $17^{\mathrm{h}} 12^{\mathrm{m}} 08^{\mathrm{s}} .3$ & $-38^{\circ} 30^{\prime} 51^{\prime \prime}$ & 13.33 & 0.2 & 78.7 & $800-460$ \\
\hline Cond 1 core & $17^{\mathrm{h}} 12^{\mathrm{m}} 08^{\mathrm{s}} .3$ & $-38^{\circ} 30^{\prime} 51^{\prime \prime}$ & 13.33 & 2.5 & 24.5 & $250-143$ \\
\hline Cond1 b & $17^{\mathrm{h}} 12^{\mathrm{m}} 10^{\mathrm{s}} .7$ & $-38^{\circ} 32^{\prime} 01^{\prime \prime}$ & 2.59 & 1.2 & 4.5 & $46-26$ \\
\hline Cond1 c & $17^{\mathrm{h}} 12^{\mathrm{m}} 08^{\mathrm{s}} .0$ & $-38^{\circ} 32^{\prime} 25^{\prime \prime}$ & 1.35 & 0.7 & 3.0 & $31-18$ \\
\hline Condensation 2 & $17^{\mathrm{h}} 12^{\mathrm{m}} 33^{\mathrm{s}} .9$ & $-38^{\circ} 30^{\prime} 49^{\prime \prime}$ & 1.92 & 0.2 & 18.8 & $192-110$ \\
\hline Cond 2 bis & $17^{\mathrm{h}} 12^{\mathrm{m}} 25^{\mathrm{s}} .9$ & $-38^{\circ} 31^{\prime} 50^{\prime \prime}$ & 0.73 & 0.2 & 5.4 & $55.5-32$ \\
\hline Condensation 3 & $17^{\mathrm{h}} 12^{\mathrm{m}} 43^{\mathrm{s}} .8$ & $-38^{\circ} 29^{\prime} 42^{\prime \prime}$ & 0.75 & 0.2 & 6.6 & $63-38$ \\
\hline Condensation 4 & $17^{\mathrm{h}} 12^{\mathrm{m}} 42^{\mathrm{s}} \cdot 3$ & $-38^{\circ} 26^{\prime} 59^{\prime \prime}$ & 0.99 & 0.2 & 8.6 & $88-50$ \\
\hline Cond 4 north & $17^{\mathrm{h}} 12^{\mathrm{m}} 46^{\mathrm{s}} .0$ & $-38^{\circ} 25^{\prime} 25^{\prime \prime}$ & 0.46 & 0.2 & 0.9 & $9-5$ \\
\hline Condensation 5 & $17^{\mathrm{h}} 12^{\mathrm{m}} 33^{\mathrm{s}} .0$ & $-38^{\circ} 20^{\prime} 00^{\prime \prime}$ & 0.77 & 0.1 & 22.1 & $226-130$ \\
\hline Cond5 a & $17^{\mathrm{h}} 12^{\mathrm{m}} 33^{\mathrm{s}} \cdot 6$ & $-38^{\circ} 19^{\prime} 55^{\prime \prime}$ & 0.77 & 0.3 & 2.6 & $26.5-15$ \\
\hline Cond5 b1+b2 $2^{2}$ & $17^{\mathrm{h}} 12^{\mathrm{m}} 41^{\mathrm{s}} .4$ & $-38^{\circ} 20^{\prime} 17^{\prime \prime}$ & 0.54 & 0.3 & 1.8 & 19-11 \\
\hline Cond5 c & $17^{\mathrm{h}} 12^{\mathrm{m}} 43^{\mathrm{s}} .3$ & $-38^{\circ} 23^{\prime} 31^{\prime \prime}$ & 0.58 & 0.3 & 1.0 & $11-6$ \\
\hline Condensation 6 & $17^{\mathrm{h}} 11^{\mathrm{m}} 49^{\mathrm{s}} .5$ & $-38^{\circ} 19^{\prime} 59^{\prime \prime}$ & 1.07 & 0.1 & 8.8 & $90-52$ \\
\hline Condensation $7^{2}$ & & & & 0.2 & 2.8 & $28.5-16$ \\
\hline Cond7 a1 & $17^{\mathrm{h}} 11^{\mathrm{m}} 59^{\mathrm{s}} .5$ & $-38^{\circ} 28^{\prime} 25^{\prime \prime}$ & 0.49 & & & \\
\hline Cond7 a2 & $17^{\mathrm{h}} 11^{\mathrm{m}} 56^{\mathrm{s}} .9$ & $-38^{\circ} 29^{\prime} 09^{\prime \prime}$ & 0.57 & & & \\
\hline Condensation 8 & $17^{\mathrm{h}} 12^{\mathrm{m}} 20^{\mathrm{s}} .0$ & $-38^{\circ} 34^{\prime} 05^{\prime \prime}$ & 0.56 & 0.2 & 3.8 & $38-22$ \\
\hline Condensation 9 & $17^{\mathrm{h}} 11^{\mathrm{m}} 54^{\mathrm{s}} .2$ & $-38^{\circ} 16^{\prime} 53^{\prime \prime}$ & 0.50 & 0.2 & 4.2 & $42-24$ \\
\hline
\end{tabular}

${ }^{1}$ Mass calculations performed with $T_{\text {dust }}=30$ and $20 \mathrm{~K}$ for the lower and higher values, respectively; ${ }^{2}$ the given flux density corresponds to the sum of the two substructures.

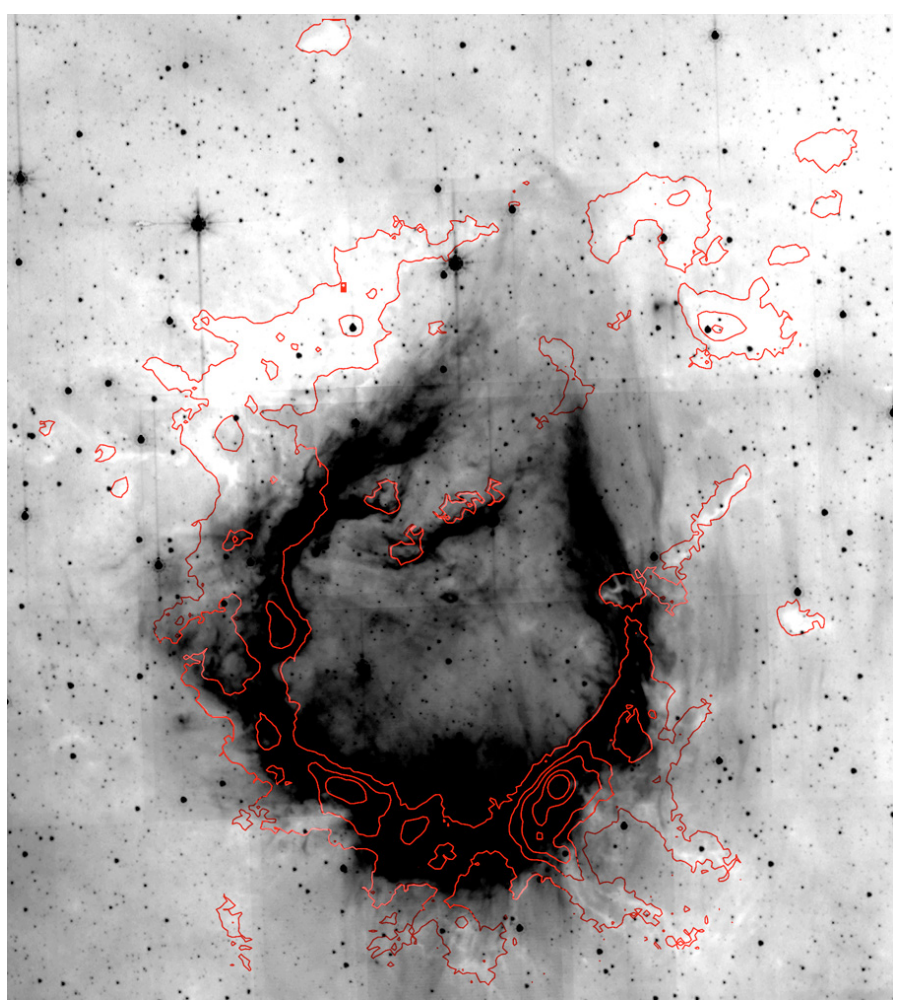

Fig. 3. Dust condensations observed at $870 \mu \mathrm{m}$ and IR dark clouds in the vicinity of RCW 120 . The red contours $(0.1,0.2,1.0,2.5$, and $5.0 \mathrm{Jy} / \mathrm{beam}$ ) correspond to the $870 \mu \mathrm{m}$ emission. The grey background is the Spitzer-GLIMPSE image at $8.0 \mu \mathrm{m}$, showing several IRDCs. The most massive condensations (in the south) are not IRDCs.

The visual extinction can be estimated from the $8.0 \mu \mathrm{m}$ absorption (cf. Vig et al. 2007), and compared with that found from the $870 \mu \mathrm{m}$ emission. The Indebetouw et al. (2005) extinction law gives $A_{8.0 \mu \mathrm{m}} / A_{K}=0.43$; the Rieke \& Lebofsky (1985) extinction law gives $A_{K} / A_{V}=0.112$. Thus $A_{V}=22.55 \tau_{8 \mu \mathrm{m}}$, with $\tau_{8 \mu \mathrm{m}}$ the optical depth at $8.0 \mu \mathrm{m}$. If $F$ and $F_{0}$ are the $8.0 \mu \mathrm{m}$ surface brightnesses, respectively in the direction of the absorbing cloud and in the direction of the surrounding background (assumed uniform), then $\tau_{8 \mu \mathrm{m}}=\ln \left(F_{0} / F\right)$. Of course, this method for deriving the extinction does not work if the absorbing structure has too great an optical thickness; an $A_{V}$ of 50 mag seems a reasonable maximum value.

Figure 4 allows us to compare these two determinations of the extinction in the direction of a zone situated north-east of RCW 120 (in the direction of condensation 5). For this region we have estimated $F_{0}$ to be equal to $45 \mathrm{MJy} / \mathrm{sr}$. The first contour, on each map, corresponds to a visual extinction of $1.7 \mathrm{mag}$. These two maps are similar, with a few differences:

- The region with $A_{V} \geq 1.7 \mathrm{mag}$ is more extended on the $8.0 \mu \mathrm{m}$ extinction map than on the $870 \mu \mathrm{m}$ map. In the low extinction zones the extinction derived from the dust emission at $870 \mu \mathrm{m}$ is $1-2 \mathrm{mag}$ less than that derived from the $8.0 \mu \mathrm{m}$ absorption. In the large extinction zones the two determinations of the extinction are in rather good agreement.

- The $8.0 \mu \mathrm{m}$ extinction map shows small-scale structures, mainly filaments and curls. In the $870 \mu \mathrm{m}$ observations these structures have been smoothed out because of the larger beam. Note that many of the large extinction filaments are perpendicular to the ionization front; we will return to this point in Sect. 5.4.

An IRDC is also observed in the $24 \mu \mathrm{m}$ image, in the same direction. Because the surrounding background is far from uniform, we did not try to estimate the extinction via the absorption at $24 \mu \mathrm{m}$.

What can we learn about the extinction from the near-IR data (from the 2MASS survey)? This data is difficult to interpret in terms of extinction. Zones devoid of stars, hence directions of large extinction, are clearly present all around RCW 120 (ZA07, Fig. 3), in the $J, H$ and $K$ maps. This is the case, for example, of the north-east large extinction zone shown in Fig. 3. Very 


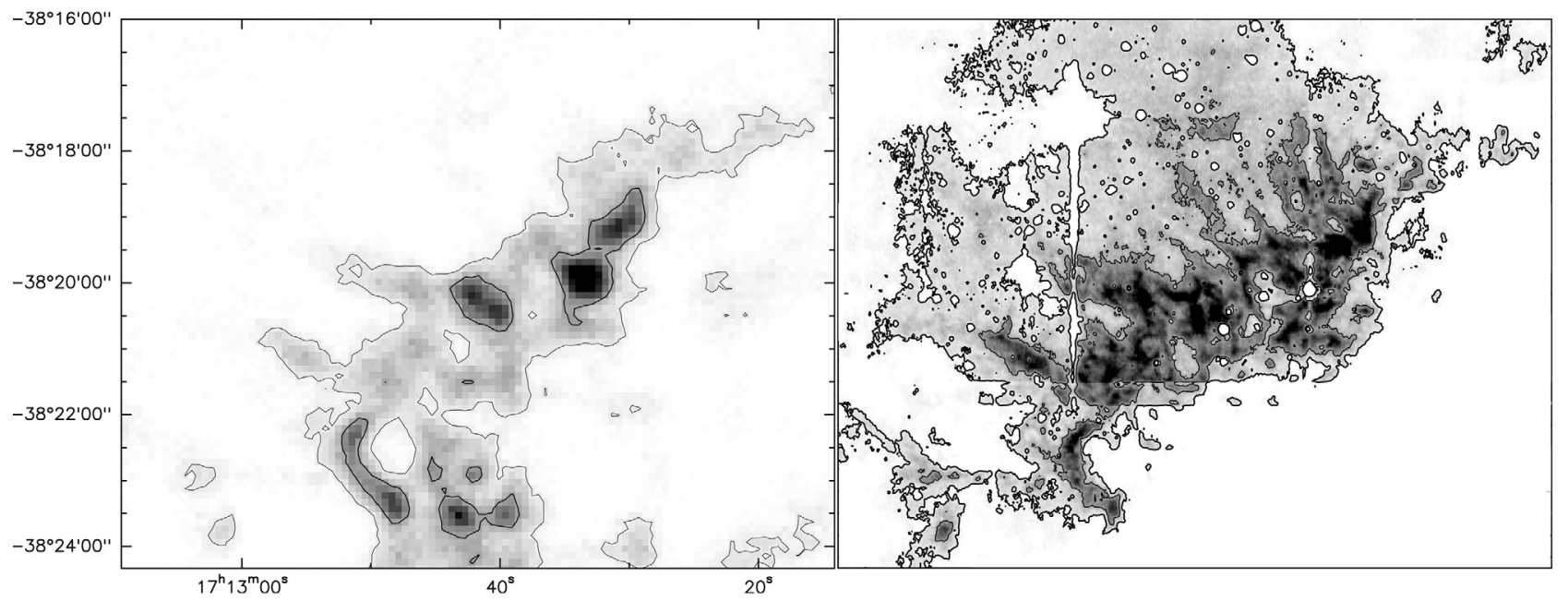

Fig. 4. Visual extinction in the direction of the north-east condensation 5. The grey scale is the same for the two figures: white is for zero local extinction, black is for an extinction of $10 \mathrm{mag}$ or larger. Left: extinction estimated from the $870 \mu \mathrm{m}$ emission. Right: extinction estimated from the $8.0 \mu \mathrm{m}$ absorption. In each figure the contours correspond to an extinction of $1.7 \mathrm{mag}$ and $5 \mathrm{mag}$.

few stars are detected towards these large-extinction filaments, whereas elsewhere numerous stars are observed, many of them displaying large extinction (up to $30 \mathrm{mag}$ in $A_{V}$ ) in the $J-H$ versus $H-K$ diagram (not shown here). These are most probably evolved stars situated in the distant background, possibly in the Galactic bulge (RCW 120 is less than $12^{\circ}$ away from the Galactic centre).

\section{Young stellar objects in the vicinity of RCW 120}

We wish to determine whether each source detected at $24 \mu \mathrm{m}$ is a star - main-sequence or evolved - or is a YSO - Class I if its luminosity is dominated by an accreting envelope or Class II if its luminosity is dominated by a disk. (Robitaille et al. 2006 prefer to describe YSOs as stage I or stage II sources.)

In the absence of a spectroscopic signature, several indicators can be used to establish the evolutionary status of a YSO, depending on the wavelength range.

- One indicator is the presence of a near-IR colour excess in the $J-H$ versus $H-K$ diagram. This excess of near-IR emission is generally attributed to hot dust associated with a disk (Lada \& Adams 1992).

- Another indicator often used with the Spitzer-GLIMPSE survey is the position of the source in the Spitzer-IRAC colourcolour diagram, [3.6]-[4.5] versus [5.8]-[8.0]. In this diagram, unreddened stars (both main-sequence and evolved) have colours near $(0,0)$, whereas Class I and Class II sources occupy different regions, as shown by Allen et al. (2004) and discussed by Whitney et al. (2003a,b, 2004).

- The slope of the spectral energy distribution (SED) between the near-IR and the mid-IR, for example from $2 \mu \mathrm{m}$ to 10 or $20 \mu \mathrm{m}$, is another indicator. This slope is defined as $\alpha=\mathrm{d}\left(\log \left(v F_{v}\right)\right) / \mathrm{d}(\log (v))$ In the following we shall consider that Class I sources have an increasing SED, i.e. $\alpha>0$, Class II sources have a decreasing SED with $-1.6 \leq \alpha \leq 0$, and Class III sources have $\alpha<-1.6$. One can use for example the $K$ versus $K-$ [24] diagram (Rebull et al. $2007^{3}$ ).

\footnotetext{
${ }^{3}$ Rebull et al. (2007) also identify "flat spectrum" sources, which are intermediate between Class I and Class II, and correspond to $-0.3<$ $\alpha<+0.3(6.75<K-[24]<8.31)$. The domain of flat spectrum sources is indicated in Fig. 5.
}

Considering sources corrected for the interstellar extinction, Class I objects have $K-[24]>7.54$, and Class II objects have $K-$ [24] between 3.37 and 7.54. Class III and stars, which we do not attempt to separate, have $K-[24]<3.37$. (Ordinary stellar photospheres have $K-[24] \sim 0$.)

We shall compare the information given by these three indicators to discuss the evolutionary stages of the sources observed at $24 \mu \mathrm{m}$ in the vicinity of RCW 120 , and listed in Table 1 . We shall show the limitations of these indicators and try new ones. We have been greatly helped in this study by the comprehensive discussion of Robitaille et al. (2006).

Figure 5 presents the $K$ versus $K$-[24] diagram obtained for the 118 sources that have $K$ and [24] magnitude measurements. We are tempted to identify 24 Class I sources (red symbols), and 51 Class II sources (green symbols) in this diagram. However, note the strong effect of interstellar extinction on the $K$-[24] colour, and the suspicious concentration of sources near the separation line between Class II and Class III objects. We shall return to this point later in this section. (Following Robitaille et al. 2006, we here refer - unconventionally - to the extinction as "interstellar" when it is due to dust located outside the envelope and/or disk of a source.)

Figure 6 presents the [3.6]-[4.5] versus [5.8]-[8.0] diagram for the 103 sources that have been measured in the four IRAC bands. We use the same symbols as in Fig. 5 for the Class I and Class II sources already identified with the first indicator ( $K$ versus $K-$ [24]). Fig. 6 shows an almost perfect agreement between the two indicators for Class I sources. Eleven new very red sources (black squares), which have no measurable $K$ magnitude and hence are absent in Fig. 5, are identified as Class I. The situation is less satisfactory for the candidate Class II sources (according to our first indicator). A number of these sources lie inside the ellipse of stars. We shall return to this point later in this section.

Figure 7 is the $J-H$ versus $H-K$ diagram for the 112 sources with measured $J, H$, and $K$ magnitudes. All the Class I sources (identified by our first indicator) exhibit near-IR excesses, and they are nearly the only ones to do so. (The relative amounts of the near-IR excesses is uncertain, as almost all these sources have uncertain 2MASS magnitudes.) The fact that most of the sources previously classified as Class II do not have near-IR excesses is surprising, since in models of Lada \& Adams (1992), 


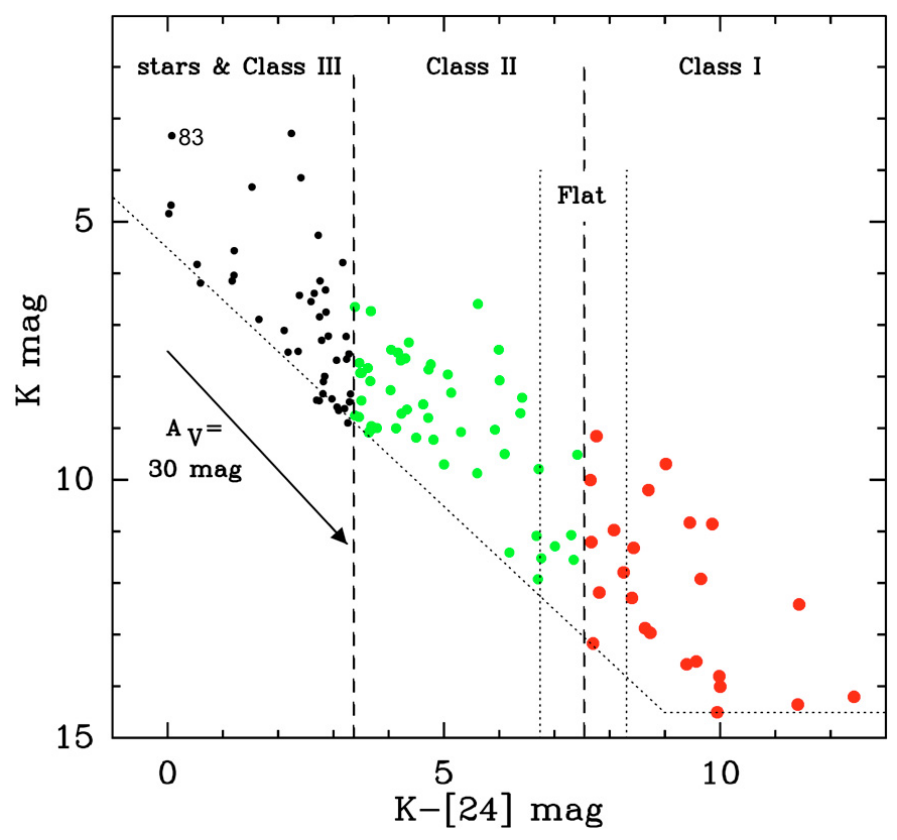

Fig. 5. The $K$ versus $K-$ [24] diagram. Different classes of sources are separated by dashed lines. The dotted lines correspond to the detection limits of $14.5 \mathrm{mag}$ in $K$ and $5.5 \mathrm{mag}$ in [24]. (Note that these detection limits are not completeness limits.) The black arrow is a reddening line corresponding to a visual extinction of $30 \mathrm{mag}$. Class I sources appear as large red dots, Class II sources as medium green dots, and all the other sources as small black dots. The same symbols will be used for the same sources in Figs. 6-8 (left).

the hot dust situated in the disk emits in the near-IR. One possible explanation is that the slope of the extinction curve is steeper than that adopted in Fig. 7. For this figure we have used the interstellar extinction law of Indebetouw et al. (2005). According to these authors, $A_{J} / A_{K}=2.50 \pm 0.15$ and $A_{H} / A_{K}=1.55 \pm 0.08$, giving a slope $\left(A_{J}-A_{H}\right) /\left(A_{H}-A_{K}\right)=1.73 \pm 0.67$. This range of values is illustrated in Fig. 7, showing that a small increase of the slope would result in a near-IR excess for numerous Class II sources.

The sources with $K-[24]<3.37$ are Class III objects or stars - in the later case mainly evolved stars. Main-sequence stars at the distance of RCW 120 or in the background (thus possibly reddened) should have $K>5.67 \mathrm{mag}$ (Martins \& Plez 2006). Thus all the sources brighter than 5.67 in $K$ and with $K-[24]<$ 3.37 are possibly giants or supergiants. This is compatible with their positions in the $J-H$ versus $H-K$ diagram. One of these sources, \#83, is very bright in $K$ and lies in the direction of the ionized gas. This star is HD 155 275, an MOIII star (Houk 1982; it is identified in Deharveng \& Zavagno 2008).

Many sources may be affected by a large interstellar extinction. Figure 7, for example, shows that many stars (black dots) or candidate Class II sources (green dots) may be affected by an (external) extinction between 20 and 40 mag. This extinction may severely affect the classification of the sources. We suggest that some stars or Class III objects, affected by strong extinction, are mistaken for Class II objects by our first indicator. Our first indicator shows a large concentration of stars and Class III and Class II objects at $K-[24] \sim 3.37$. All the Class II sources which have $3.37<K-[24]<4.0$ lie inside the ellipse (showing the location of stars) in Fig. 6. Furthermore, according to their $J-H$ and $H-K$ colours (Fig. 7), all these sources may be evolved stars. Thus most of these stars are probably evolved background stars and not Class II sources.

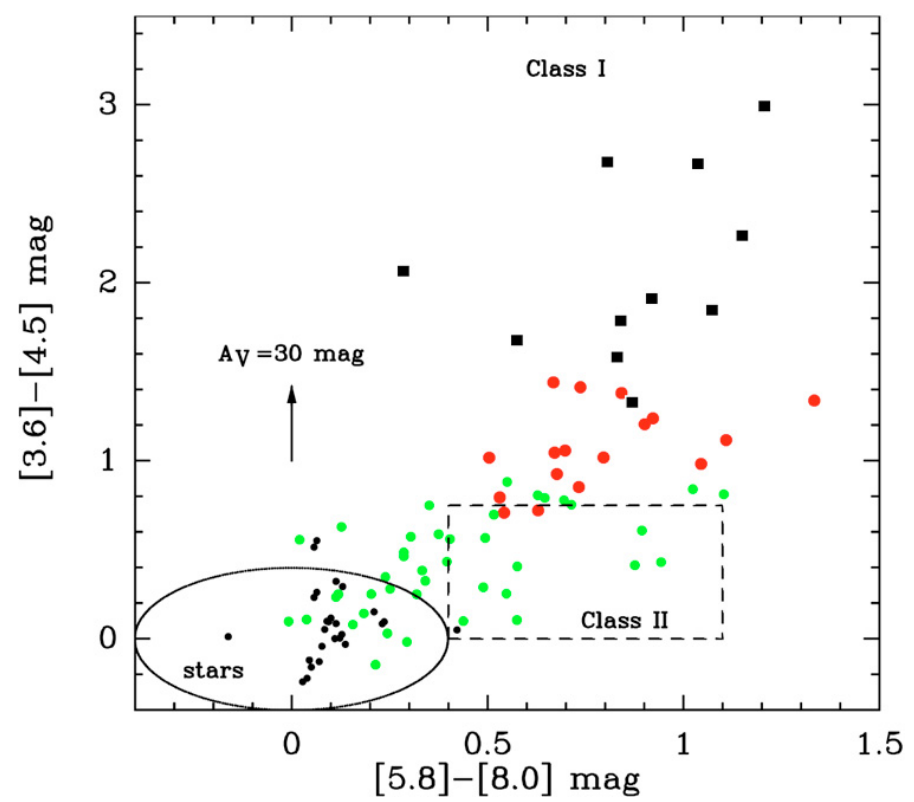

Fig. 6. The [3.6]-[4.5] versus [5.8]-[8.0] diagram. The black arrow is a reddening line corresponding to a visual extinction of 30 mag. Each source is represented by the same symbol as in Fig. 5. The black squares are for sources lacking $K$ magnitudes. The locations, according to Allen et al. (2004), of sources dominated by their photospheres (stars and Class III objects) and of Class II sources are indicated by an ellipse and a rectangle, respectively.

And the situation is perhaps not better for Class I sources. Fig. 8 presents the [24] versus [8]-[24] diagram, very similar to the $K$ versus $K-[24]$ diagram of Fig. 5, with the disadvantage of a smaller wavelength range but the advantage of a weaker influence of the extinction. In this diagram unreddened Class I sources have [8]-[24] > 3.58, and Class II sources have $1.67 \leq[8]-[24] \leq 3.58$. We have kept the same symbols as given by our first indicator. Figure 8 (left) shows that some sources previously classified as Class I now appear as Class II, and that some sources previously classified as Class II are probably Class III sources or stars.

We shall now try to estimate the possible influence of the interstellar extinction on our classifications. We use the $870 \mu \mathrm{m}$ emission to derive a maximum value of this external extinction; we assume that the sources lie in the centres of the dust condensations observed at $870 \mu \mathrm{m}$ in their direction. The extinction is then given by

$A_{V}($ external $)=\frac{16.75 F_{870 \mu \mathrm{m}}}{2}+3$

where $F_{870 \mu \mathrm{m}}$ is expressed in $\mathrm{Jy} /$ beam and the 3 is to correct for a 3 mag underestimate of the extinction using the $870 \mu \mathrm{m}$ map (Sect. 3.2). Figure 8 (right) gives an idea of the maximum external extinction possibly affecting the sources. It shows that only a few sources are possibly misclassified for this reason. These are mainly sources observed in the direction of the bright condensation 1 (\#41, \#44, \#46, \#47, \#49, \#54) and in condensation 6 (\#106, \#110) (Sect. 5.3). These sources may be flat spectrum sources. For the other sources, the discrepancy between the classifications given by different indicators is most probably due to difficult photometric measurements. It mainly affects sources lying near the separation limits of the different classes.

Table 1 gives our conclusions concerning the evolutionary stages of the sources. As Class I we include 21 sources satisfying all the indicators ( $K$ versus $K-[24],[3.6]-[4.5]$ 


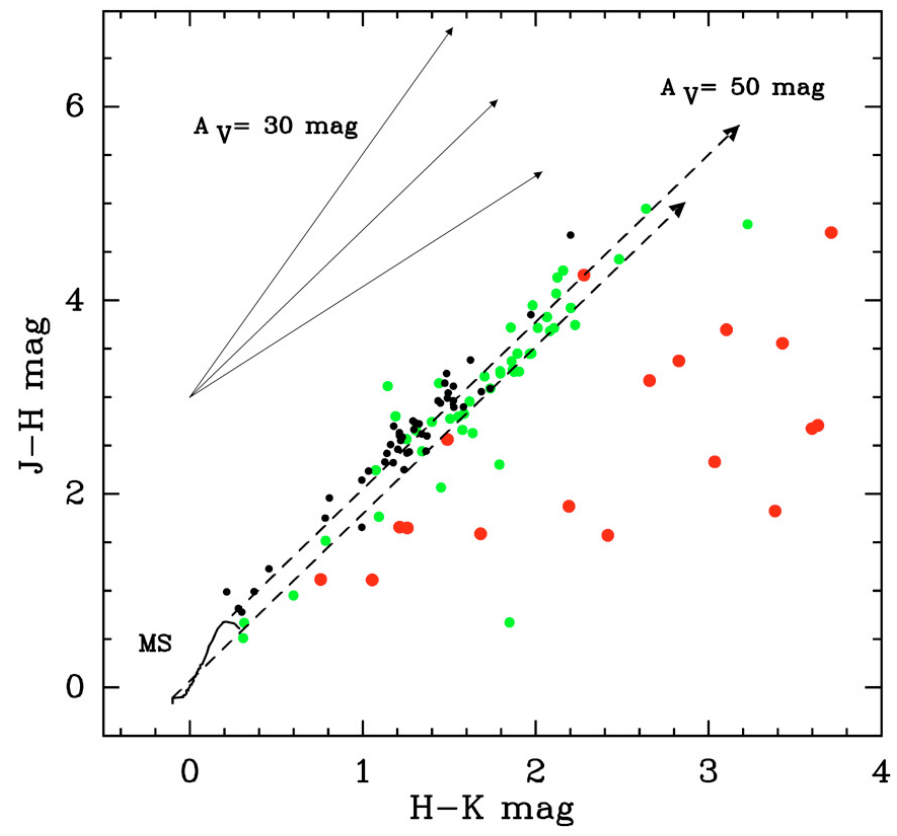

Fig. 7. The $J-H$ versus $H-K$ diagram. The main sequence (MS) is drawn with a solid curve; the colours are from Martins \& Plez (2006) for O stars, and from Tokunaga (2000) for later spectral types. The reddening lines (dashed lines), for $\mathrm{O} 5 \mathrm{~V}$ and $\mathrm{M} 2 \mathrm{~V}$ stars, are for a visual extinction of 50 mag (extinction law of Indebetouw et al. 2005). The symbols are the same as in Fig. 5. All the sources situated below the lower (O-star) reddening line exhibit a near-IR excess. The sources situated above the upper reddening line are possibly evolved stars. The solidline arrows are the reddening lines corresponding to a visual extinction of 30 mag and compatible with the extinction law of Indebetouw et al. (2005, see text).

versus [5.8]-[8.0], [24] versus [8]-[24]) and not affected by too much external extinction. We consider as intermediate Class I-Class II 18 sources which are Class I or Class II depending on the indicator (identified by "I-II?" in Col. 13 of Table 1), or are possibly flat spectrum sources mistaken for Class I because of a large external extinction ("I-flat?" in Table 1)). We consider as Class II 29 sources satisfying all the indicators, and as intermediate Class II-Class III 9 sources which are Class II or Class III depending on the indicators ("II-III?" in Table1). Note that in Table 1 we have classified as "stars" all the sources dominated by their photosphere, and which we are unable to distinguish: Class III YSOs, MS or evolved stars.

The spatial distribution of the YSOs is presented on Fig. 9 and is discussed in Sect. 5.3.

\section{Discussion}

\subsection{Dust condensations and infrared dark clouds}

RCW 120 demonstrates in an exemplary way that massive dust condensations are not all infrared dark clouds. A dense neutral condensation needs to be seen towards a bright background emission to appear as an IRDC. But another necessary condition is to not be located behind bright foreground emission. In the case of RCW 120 the structures that are the most massive (condensations 1 and 2) and the densest (the core in condensation 1, responsible for an extinction $\sim 225 \mathrm{mag}$ ) are not IRDCs: they are masked by the bright emission of the adjacent foreground PDR. Only their external parts (those far from the IF) appear as faint absorption features (Fig. 3).

\subsection{Young stellar objects and AGB stars}

Robitaille et al. (2008) present a catalogue of intrinsically red sources, observed by Spitzer, in the Galactic mid-plane. These sources consist mostly of high- and intermediate-mass YSOs and asymptotic giant branch (AGB) stars. As shown by these authors, it is very difficult to distinguish between these two types.

In the following we compare the sources detected at $24 \mu \mathrm{m}$ in the vicinity of RCW 120 with the red sources selected by Robitaille et al. If we apply their selection criteria, i.e. $[4.5]-[8.0] \geq 1,13.89 \geq[4.5] \geq 6.5$, and $9.52 \geq[8.0] \geq 4.01$, to the 112 sources with [4.5] and [8.0] magnitudes in Table 1, we find 39 sources; 26 of them are Class I or flat spectrum sources, 11 are Class II, and two more are uncertain Class II-Class III.

Robitaille et al. estimate that $\sim 19$ percent of the red sources may be AGBs. If true, this means that up to seven of our 39 red sources may be AGBs and not YSOs! It is very difficult to distinguish between these two categories if we only have photometric measurements up to $24 \mu \mathrm{m}$. Robitaille et al. show that YSOs are generally redder than AGBs between $8 \mu \mathrm{m}$ and $24 \mu \mathrm{m}$, and propose to use the criterion [8.0]-[24] $\geq 2.5$ to identify YSOs. Using this criterion we find that 32 of our 39 red sources are probably YSOs (the seven sources with [8.0]-[24] $\leq 2.5$ are Class II or uncertain Class II). Thus we must keep in mind in the following that a few of our sources identified as YSOs, and especially Class II YSOs, are possibly AGB stars and are therefore evolved.

\subsection{Large scale distribution of the YSOs}

We have measured 138 sources around RCW 120 emitting at $24 \mu \mathrm{m}$. Three are unclassified. Twenty-one are Class I YSOs, and eighteen are possible Class I or flat spectrum sources. Twentynine sources are Class II YSOs, and nine sources are more uncertain Class II sources. Thus 49 to 56 percent of the $24 \mu \mathrm{m}$ sources are young stellar objects, 28 percent of the $24 \mu \mathrm{m}$ sources being Class I or flat spectrum sources.

Figure 9 shows the large-scale distribution of the YSOs possibly associated with RCW 120. It shows a large number of Class I or flat spectrum sources (red symbols), all but five associated with the shell of dense neutral material surrounding RCW 120. Thus even if we have no direct proof that any given YSO is associated with RCW 120, this general picture demonstrates that most of them are associated with the $\mathrm{H}$ II region, and that their formation has been triggered, one way or another, by the H II region. On the other hand, the Class II sources are more widely scattered over the field. Their association with RCW 120 is less evident, and this raises questions about their origin.

We note the large concentration of Class I or flat spectrum YSOs in the direction of condensation 1, the most massive.

\subsection{Stellar content of the condensations}

In the following we discuss the YSO content of a few condensations.

- Condensation 1: this is the most massive of the condensations, in the range $460-800 M_{\odot}$. It contains a very massive core, of 140-250 $M_{\odot}$, and several other bright substructures. Figure 10 shows this condensation and the numerous $24 \mu \mathrm{m}$ sources observed in its direction or vicinity.

The bright $870 \mu \mathrm{m}$ core has a size (beam-corrected FWHM) of 14 ". $6 \times 7$ ". $7(0.095 \mathrm{pc} \times 0.05 \mathrm{pc})$, along the IF and perpendicular to it, respectively - thus slightly elongated along the IF. Its size and peak flux indicate a mean density of a few 

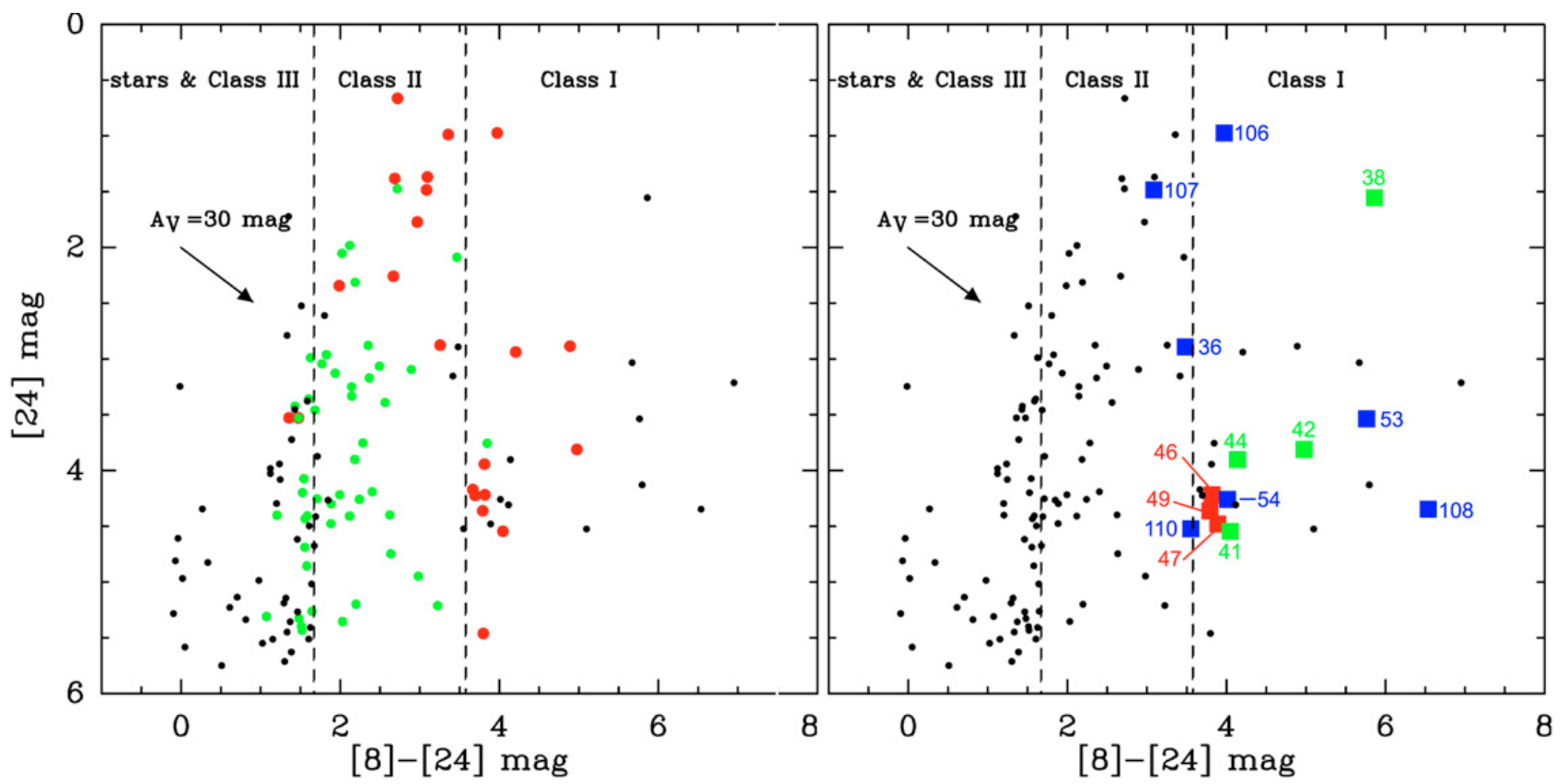

Fig. 8. The [24] versus [8]-[24] diagram, with the different classes separated by dashed lines. Class I sources have [8]-[24] $\geq 3.58$, Class II sources have $1.67 \leq[8]-[24] \leq 3.58$. The reddening vectors are for a visual extinction of $30 \mathrm{mag}$. Left: the red and green dots correspond to Class I and Class II sources, according to our first indicator. Right: the maximum external extinction possibly affecting the sources is indicated as follows: red squares are for sources with $A_{V}$ (external) $\geq 30 \mathrm{mag}$, green squares for sources with $20 \mathrm{mag} \leq A_{V}$ (external) $\leq 30$ mag, blue squares for sources with $5 \mathrm{mag} \leq A_{V}$ (external) $\leq 10 \mathrm{mag}$ (no sources with $10 \mathrm{mag} \leq A_{V}$ (external) $\leq 20$ mag are present in this diagram).

times $10^{6}$ molecules $\mathrm{cm}^{-3}$. No bright $24 \mu \mathrm{m}$ source is observed in the direction of the $870 \mu \mathrm{m}$ emission peak, but three faint sources (including \#47) may be associated with it. The SpitzerMIPSGAL image at $70 \mu \mathrm{m}$ in Fig. 11 shows an enhancement of the emission in the direction of the core, suggesting that a very deeply embedded object is present here. We measured a flux of $130 \mathrm{Jy}$ for this object, but this is very uncertain due to the underlying emission of the PDR and the low quality of the $70 \mu \mathrm{m}$ image.

According to our indicators, many $24 \mu \mathrm{m}$ sources appear to be Class I YSOs. Among these are \#38, which is observed in the direction of a spherical dust clump (b in Table 2 and Fig. 10); \#58, also observed on the border of clump a 2 in condensation 7; and \#61, observed in the direction of the ionized gas (but most probably situated behind the H II region). Sources \#36 and \#37, also located in projection close to a condensation, are possibly in an intermediate stage between Class I and Class II. The stage of \#39 depends on the indicators used, but is probably a Class II. Source \#32 has only [4.5] and [24] magnitudes; the slope of its SED suggests a Class I source.

Condensation 1 contains a most remarkable feature: a chain of eleven $24 \mu \mathrm{m}$ sources. They are aligned parallel to the main IF and are regularly spaced, separated by some $0.1 \mathrm{pc}$. They are deeply embedded. Sources \#42 and \#53 are Class I. Sources \#41, $\# 44, \# 46$, \#47, \#49, and \#54 lie near the separation between Class I and Class II and may be flat spectrum sources as they may be affected by a large external extinction (cf. Fig. 8). Measurements lack for \#40, \#48, and \#52; the slopes of their SEDs suggest Class I sources.

We have fitted the SEDs of a few sources using the Webbased SED fitting-tool of Robitaille et al. (2007, http:// caravan.astro.wisc.edu/protostars/). The parameters of the best model, as well as a range of possible values for some parameters, are given in Table 3. YSO \#38 is a stage I source, with a central object of a few solar masses; condensation $b$ is probably not the source's cold envelope since its flux density at $870 \mu \mathrm{m}$ is too large. Source \#61 is also in stage I, with a central object of a few tenths of a solar mass. For sources \#53 and \#49, most of the models indicate that they are stage I sources around central objects of about one solar mass, and with massive disks; but a few of the good models (those with $\chi^{2}-\chi_{\text {best }}^{2} \leq 3$ ) show central objects with a higher mass, no accreting envelopes, and massive disks, hence corresponding to stage II sources.

The chain of sources aligned along the IF, and regularly spaced, indicates that star formation has occurred in the collected layer, probably resulting from gravitational instabilities along a dense filament. At a temperature of $20 \mathrm{~K}$ and a density of $10^{5}$ molecules per $\mathrm{cm}^{3}$, gravitational instabilities form cores separated by the Jeans length $\lambda_{\mathrm{J}} \sim 0.1 \mathrm{pc}$, and with the Jeans mass $M_{\mathrm{J}} \sim 1.5 M_{\odot}$. This is probably what has occurred inside condensation 1 . On the other hand the formation of the very massive and dense core may result from the collect and collapse process, i.e. from large-scale gravitational instabilities along the surface of the collected shell. Here again triggered star formation is probably at work, as shown by the presence of a very deeply embedded source - hence a Class 0 YSO - only detected at $70 \mu \mathrm{m}$.

- Condensation 2: this lies at the south-east border of RCW 120, adjacent to the main IF. Its mass is in the range 110-290 $M_{\odot}$ (not including the elongated structure which we call condensation 2 bis in Fig. 12). Its peak column density corresponds to a visual extinction $\sim 32 \mathrm{mag}$. The YSO \#50 lies at less than $0.03 \mathrm{pc}$ (in projection) from the peak direction.

This source has no near-IR emission (in the 2MASS survey), and no measured $8.0 \mu \mathrm{m}$ flux, hence does not appear in our diagrams. However the slope of its SED between $3.6 \mu \mathrm{m}$ and $24 \mu \mathrm{m}$ is between 2.0 (without extinction correction) and 1.3 (correcting for a visual extinction of $32 \mathrm{mag}$ ). Thus source \#50 is a Class I object. It lies very close to the IF, which is probably the reason why we do not detect it at $70 \mu \mathrm{m}$. 


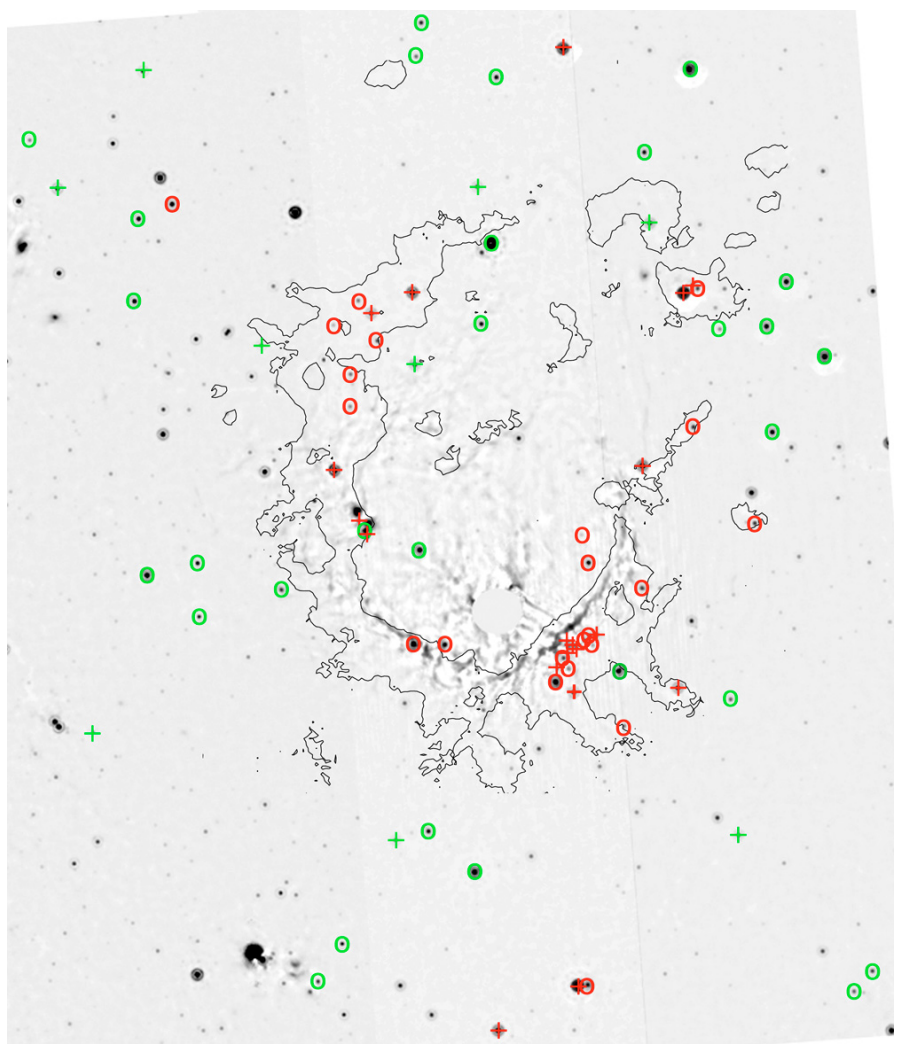

Fig. 9. Distribution of the Class I (red circles), intermediate Class I-Class II or flat spectrum (red crosses), Class II (green circles), and possible Class II-Class III sources (green crosses). The grey-scale background is an unsharp-masked $24 \mu \mathrm{m}$ image, where the extended dust emission has been subtracted to enhance the stellar component. The contours represent the $870 \mu \mathrm{m}$ emission level of $0.1 \mathrm{Jy} / \mathrm{beam}(5 \sigma)$.

Source \#51 lies at the top of a "finger", a structure of the IF protruding inside the ionized gas, and pointing towards the exciting star. This finger, which is seen south-west of condensation 2, is an elongatd dust filament. Another such structure lies nearby, which we have called condensation 2bis (Fig. 12). ZA07 proposed that these structures result from dynamical instabilities of the expanding IF, such as these simulated by García-Segura \& Franco (1996). These structures are found towards a gap between condensations 1 and 2, through which the ionized gas tries to flow away from the central $\mathrm{H}$ II region (a region thus dynamically unstable). An alternative origin is possible. These two "fingers" are reminescent of the "pillars" in M 16, with Class I \& II YSOs located at their tops (Sugitani et al. 2002; Indebetouw et al. 2007, and references therein). The pillars in M 16 clearly result from the interaction of NGC 6611's OB stars' UV radiation with pre-existing dense clumps. However, as shown by the molecular observations of White et al. (1999), most of the mass of the pillars is located in their heads rather than their tails, whereas the contrary is observed in RCW 120's fingers (at least in condensation 2bis). Thus the origin of the fingers and of YSO \#51 is uncertain; higher angular resolution observations are needed.

Our indicators show that YSO \#51 is possibly a Class I object. Its SED, modelled by the Robitaille et al. fitting tool, confirms this conclusion. The best model indicates a central object of $1.3 M_{\odot}$ with a massive disk but, however, with an accreting envelope, hence a stage I-stage II object. Several parameters are rather uncertain, but the disk is massive in all the models (Table 3).

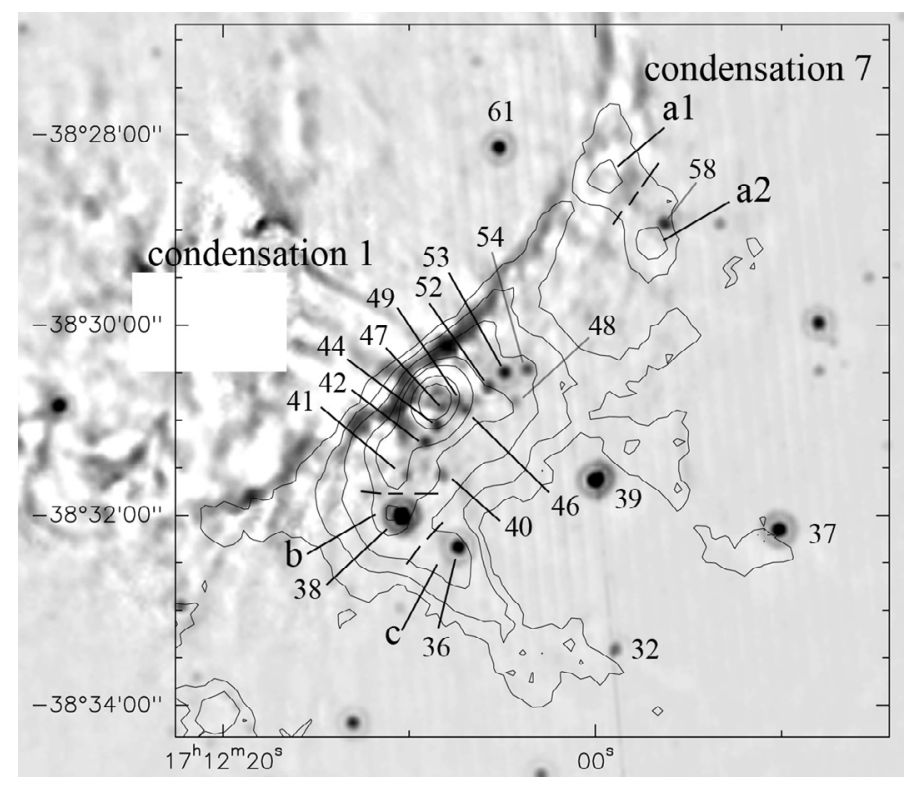

Fig. 10. Condensations 1 and 7: the background image is an unsharp masked image obtained from the Spitzer-MIPSGAL frame at $24 \mu \mathrm{m}$. This image, free of the diffuse extended emission, enhances all the stellar sources. The contours correspond to the $870 \mu \mathrm{m}$ emission. The contour levels are $0.2 \mathrm{Jy} / \mathrm{beam}$ (used to estimate the masses of condensations 1 and 7), 0.4, 0.8, 1.75, 2.5 (used to estimate the mass of the core in condensation 1), 5, and $8 \mathrm{Jy} /$ beam.

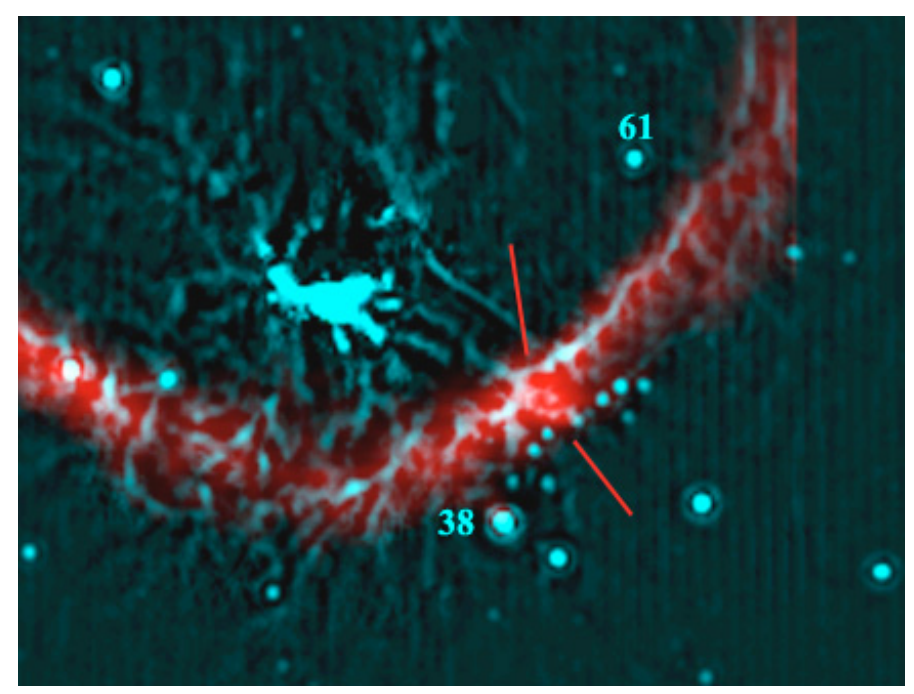

Fig. 11. Colour composite image of the southern IF in the mid-IR. The Spitzer-MIPS emission at $70 \mu \mathrm{m}$ appears in red. Turquoise shows an unsharp-masked image at $24 \mu \mathrm{m}$, obtained from Spitzer-MIPSGAL. The red lines point to the $70 \mu \mathrm{m}$ source detected in the exact direction of the dense core in condensation 1.

Finally the other YSO of the field, \#59, is clearly a rather massive stage II-stage III source. It is well fitted by a central object of $6.6 M_{\odot}$, a small disk, and no envelope (Table 3). Its association with RCW 120 is uncertain.

- Condensation 4: this is elongated along the IF but protrudes inside the ionized region. Its mass is between 50 and $90 M_{\odot}$. Figure 13 shows that several $24 \mu \mathrm{m}$ sources are detected in its vicinity, but that none is observed in the exact direction of the $870 \mu \mathrm{m}$ emission peak. However all three sources (\#64, $\# 67$, and \#69) lie on the border of the condensation facing the ionized gas and have near-IR counterparts. Our indicators show 
Table 3. Parameters of the "best fit models" for the SEDs of a few sources, obtained using the SED fitting tool of Robitaille et al. (2007).

\begin{tabular}{ccrcccc}
\hline \hline YSO & $\begin{array}{c}M_{\text {star }} \\
\left(M_{\odot}\right)\end{array}$ & $\begin{array}{r}T_{\text {star }} \\
(\mathrm{K})\end{array}$ & $\begin{array}{c}M_{\text {disk }} \\
\left(M_{\odot}\right)\end{array}$ & $\begin{array}{c}\dot{M}_{\text {envelope }} \\
\left(M_{\odot} \mathrm{yr}^{-1}\right)\end{array}$ & $\begin{array}{c}L \\
\left(L_{\odot}\right)\end{array}$ & Comment \\
\hline$\# 38$ & 3.2 & 4350 & $2.4 \times 10^{-3}\left(10^{-3}-10^{-1}\right)$ & $1.7 \times 10^{-4}$ & 98 & stage I \\
$\# 61$ & 0.3 & 3370 & $4.3 \times 10^{-2}$ & $7.3 \times 10^{-5}$ & 20 & stage I \\
$\# 51$ & 1.3 & 4355 & $6.3 \times 10^{-2}$ & $1.810^{-6}\left(10^{-6}-10^{-4}\right)$ & 17 & stage I-II \\
$\# 59$ & 6.6 & 19700 & $1.0 \times 10^{-5}\left(10^{-5}-10^{-4}\right)$ & 0 & 1320 & stage II-III \\
$\# 67$ & 5.1 & 7554 & $1.7 \times 10^{-3}\left(10^{-5}-10^{-1}\right)$ & $2.2 \times 10^{-7}$ & 280 & stage II \\
$\# 88$ & 4.1 & 4400 & $4.4 \times 10^{-2}\left(10^{-3}-10^{-1}\right)$ & $3 \times 10^{-4}$ & 130 & stage I \\
$\# 105$ & 1.7 & 4200 & $4.5 \times 10^{-2}\left(10^{-3}-10^{-2}\right)$ & $6 \times 10^{-4}$ & 38 & stage I \\
$\# 106$ & 2.0 & 4160 & $2.2 \times 10^{-1}$ & $1.2 \times 10^{-5}$ & 105 & uncertain \\
\hline
\end{tabular}

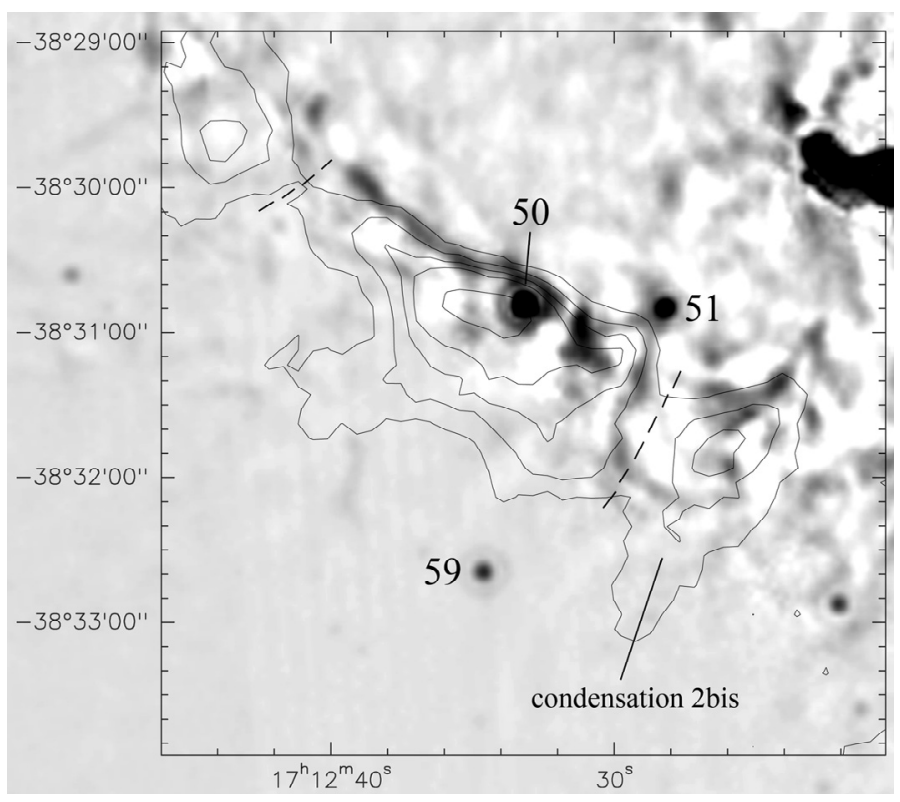

Fig. 12. Condensation 2: the background image is the unsharp masked image at $24 \mu \mathrm{m}$; the contours correspond to the $870 \mu \mathrm{m}$ emission. The contour levels go from $0.2 \mathrm{Jy} / \mathrm{beam}$ (used to estimate the masses of the condensations) to $1.8 \mathrm{Jy} /$ beam, by step of $0.2 \mathrm{Jy} /$ beam. The dashed lines show the north-east and south-west limits adopted to delimit condensation 2 . The nearby condensation 2 bis is identified.

that \#64 is intermediate between Class I and Class II, and that \#67 is a Class II. Source \#69 has no measurement at $8.0 \mu \mathrm{m}$, so it is not in our diagrams, but the slope of its SED between $3.6 \mu \mathrm{m}$ and $24 \mu \mathrm{m}$ lies between 0.4 (uncorrected for extinction) and 0.1 (assuming $A_{V}=14 \mathrm{mag}$, corresponding to the $870 \mu \mathrm{m}$ column density in its direction); thus source \#69 is probably intermediate between Class I and Class II.

We have used the Robitaille et al. SED fitting tool to model source \#67. The result confirms our previous conclusion. The parameters of the best model are given in Table 3. This source contains a central object of mass $\sim 5 M_{\odot}$. Many of the good models have no accretion from an envelope. Thus this source is most probably a stage II, but the mass of the disk is very uncertain.

Two regions of extended emission at $8.0 \mu \mathrm{m}$ also lie on the border of condensation 4. ZA07 suggested that they were PDRs around Herbig $\mathrm{Ae} / \mathrm{Be}$ stars. Emission from these PDRs is also observed at $24 \mu \mathrm{m}$. Their central stars have been observed by 2MASS; their near-IR photometry suggests a B4V star affected by a visual extinction of 7.8 mag (object $\mathrm{A}$ in Fig. 13, or object 1 in ZA07), and a B7V star with an extinction of 12.2 mag (object B in Fig. 13, or object 2 in ZA07). These stars are

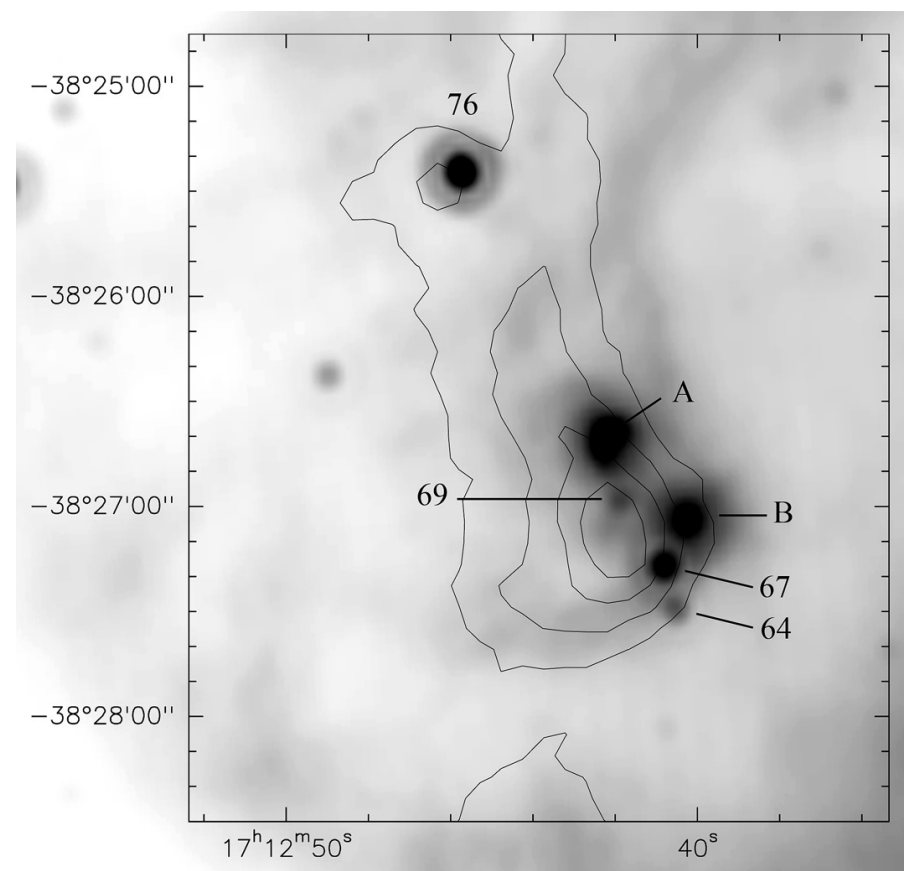

Fig. 13. Condensation 4: the background image is the SpitzerMIPSGAL image at $24 \mu \mathrm{m}$; the contours show the $870 \mu \mathrm{m}$ emission. The contour levels are $0.2 \mathrm{Jy} /$ beam (used to estimate the mass of the condensation), $0.4,0.6$, and $0.8 \mathrm{Jy} /$ beam.

probably the most massive of the sources associated with condensation 4.

ZA07 proposed that condensation 4 was a pre-existing dense clump which became surrounded by ionized gas during the expansion of the HII region. Star formation is observed at its borders and not at its centre. Thus star formation is probably not due to the clump implosion (which is predicted by the radiation-driven implosion models; Lefloch \& Lazareff 1994; Kessel-Deynet \& Burkert 2003).

Figure 13 shows the presence of a small spherical clump north of condensation 4 , with a mass in the range 5-9 $M_{\odot}$. A bright $24 \mu \mathrm{m}$ YSO, \#76, coincides with this clump to within the uncertainties (about $0.015 \mathrm{pc}$ in projection). The evolutionary stage of \#76 is uncertain: our indicators point to Class I or Class II. But most of the SED models have no accreting envelope, suggesting stage II.

- Condensation 5: this is a group of structures emitting at $870 \mu \mathrm{m}$ on the north-east border of RCW 120 (Fig. 14). Its mass is in the range $130-225 M_{\odot}$. It contains several small spherical dust clumps in the direction of which YSOs are observed: clump a, of mass $15-26 M_{\odot}$, possibly associated with \#107; 


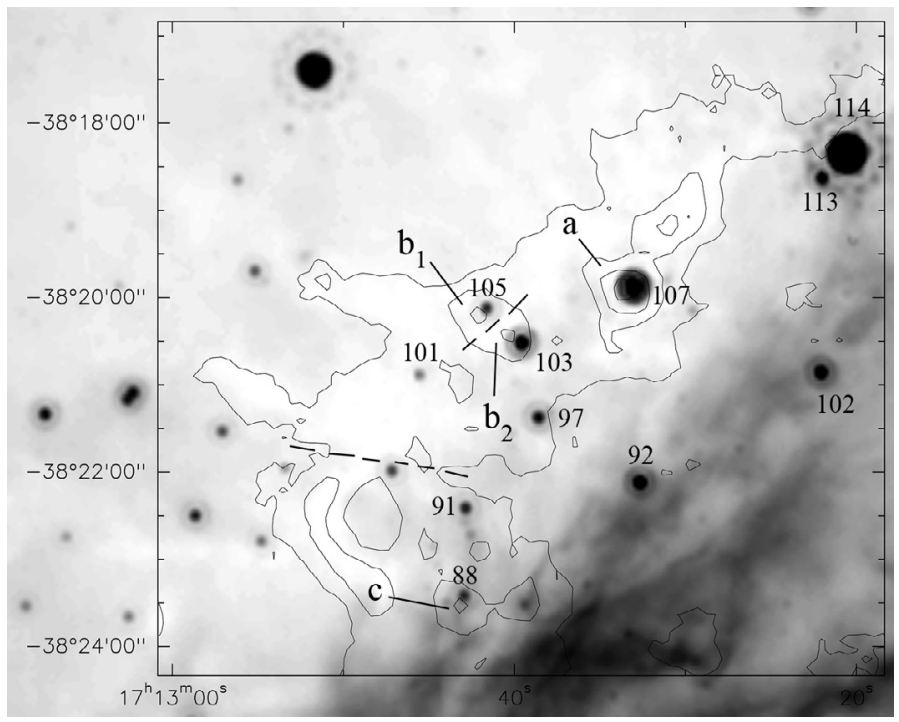

Fig. 14. Condensation 5: the background image is the Spitzer-MIPSGAL frame at $24 \mu \mathrm{m}$; the contours correspond to the $870 \mu \mathrm{m}$ emission. The contour levels are $0.1 \mathrm{Jy} /$ beam (used to estimate the mass of the condensation), $0.3,0.5$, and $0.7 \mathrm{Jy} /$ beam; the dashed line shows the adopted southern limit of the condensation.

clumps b1 + b2, of total mass 11-19 $M_{\odot}$, possibly associated with \#105 and \#103. Clump c, of mass $6-11 M_{\odot}$, possibly associated with \#88, lies outside condensation 5.

Numerous $24 \mu \mathrm{m}$ sources lie in the field of Fig. 14. According to our indicators, sources \#88, \#91 and \#97 are Class I YSOs; sources \#103 and \#107 are probably intermediate between Class I and Class II; \#102 is a Class II; and \#92 is intermediate between Class II and Class III. Magnitudes are missing for three sources; we have used the slope of the SED between $3.6 \mu \mathrm{m}$ and $24 \mu \mathrm{m}$ to classify them. Sources \#101 and \#105 have strongly rising SEDs (slopes 1.9 and 1.6 respectively) characteristic of Class I. Source \#114 is a Class II source (decreasing slope of -1.15$)$. The nature of \#113 is very uncertain, probably because it lies too close to the very bright \#114.

We have used the Robitaille et al. SED fitting tool to model the SEDs of \#88 and \#105. The parameters of the best fit model are given in Table 3. These two sources are rather similar, in an evolutionary stage I, with a massive disk. We tried to model \#103 and \#107 but the results are very uncertain (both stage I and stage II are obtained); far-IR measurements are clearly needed to constrain their SEDs.

- Condensation 6: this lies on the north-west border of RCW 120, apparently far from the main IF. However a northsouth $24 \mu \mathrm{m}$ filament, probably tracing the emission of dust in the PDR, is seen adjacent to this condensation (Fig. 15). Also, diffuse $\mathrm{H} \alpha$ emission is observed nearby (Fig. 2). Thus this condensation is submitted to the pressure of the ionized gas. Its mass is in the range 52-90 $M_{\odot}$; its peak column density corresponds to a visual extinction of $18 \mathrm{mag}$. It appears as an IRDC on both the $8.0 \mu \mathrm{m}$ and $24 \mu \mathrm{m}$ images.

Figure 15 shows the $870 \mu \mathrm{m}$ emission contours superimposed on the $24 \mu \mathrm{m}$ image. Condensation 6 contains a small cluster of at least three YSOs, separated by $\sim 0.17 \mathrm{pc}$ (in projection). YSO \#106, very bright at $24 \mu \mathrm{m}$ (possibly saturated), is also detected at $70 \mu \mathrm{m}$; it lies $0.08 \mathrm{pc}$ away from the dust emission peak. All the indicators show that these three YSOs are Class I (\#108) or flat spectrum sources (\#106, \#110).

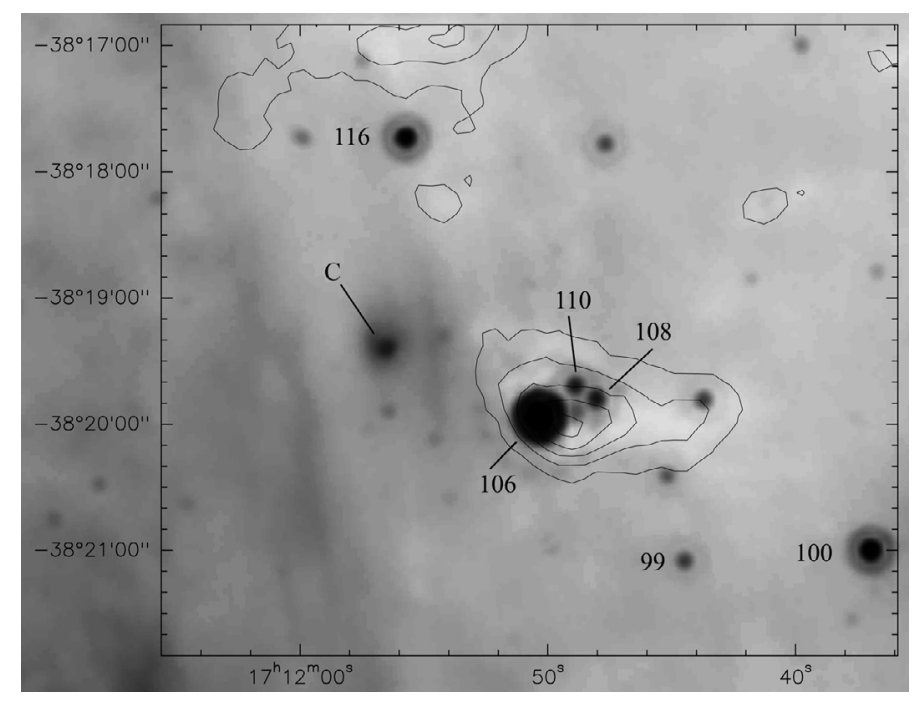

Fig. 15. Condensation 6 and its surrounding. The background image is the Spitzer-MIPSGAL frame at $24 \mu \mathrm{m}$. The contours correspond to the $870 \mu \mathrm{m}$ emission. The contour levels are $0.2 \mathrm{Jy} /$ beam (used to estimate the mass of the condensation), 0.4, 0.6, 0.8, and $1.0 \mathrm{Jy} / \mathrm{beam}$.

We tried to model the SED of object \#106 using the Robitaille et al. fitting tool. The evolutionary stage of this source is uncertain: the best model, with a central object of mass $2 M_{\odot}$ and an accreting envelope, corresponds to stage I (Table 3), but other good models have more massive central objects and no accreting envelopes, and thus correspond to stage II sources.

The other stars in the field of Fig. 15 are Class II (\#99, \#100) or intermediate Class II-Class III (\#116). Figure 15 also shows a small faint region of $24 \mu \mathrm{m}$ extended emission which we call region C. Its central star has 2MASS magnitudes $(J=12.70$, $H=11.99, K=11.65$ ) corresponding to a B9V star with a visual extinction of the order of $6.1 \mathrm{mag}$. We suggest that the extended $24 \mu \mathrm{m}$ emission comes from a PDR excited by this star. Region $\mathrm{C}$ and its exciting star are very similar to regions $\mathrm{A}$ and $\mathrm{B}$ in condensation 4 , which we believe to be second-generation objects.

\subsection{The structure of the PDR and the long-distance influence of the $H$ II region}

At first glance RCW 120 appears to be a perfect spherical bubble. However, as stressed by ZA07, the ionized gas is trying to flow out of the bubble, and consequently the H II region is elongated towards the north, where the surrounding interstellar medium is of lower density. This morphology is delineated by the shape of the ionization front and by that of the low intensity $870 \mu \mathrm{m}$ emission zones, as shown in Fig. 16, a composite image of the $870 \mu \mathrm{m}$ emission in turquoise and of an unsharp-masked image at $24 \mu \mathrm{m}$ in red. The unsharp masking emphasizes the small-scale structures of the $24 \mu \mathrm{m}$ emission, both in the ionized gas and in the PDR. The black arrows at the top of Fig. 16 point to low intensity $870 \mu \mathrm{m}$ emission zones which close the northern extension of the ionized bubble; these zones are associated with $24 \mu \mathrm{m}$ filaments in the adjacent PDR. The upper arrow shows that a low intensity $870 \mu \mathrm{m}$ emission region joins condensation 5 and condensation 9. Here again we are observing the layer of neutral material collected around the ionized region.

Figure 17 shows the extent of the PDR. This is a colour composite image of the $\mathrm{H} \alpha$ emission, in red, and of the unsharpmasked image at $24 \mu \mathrm{m}$ in turquoise. We see in red the 


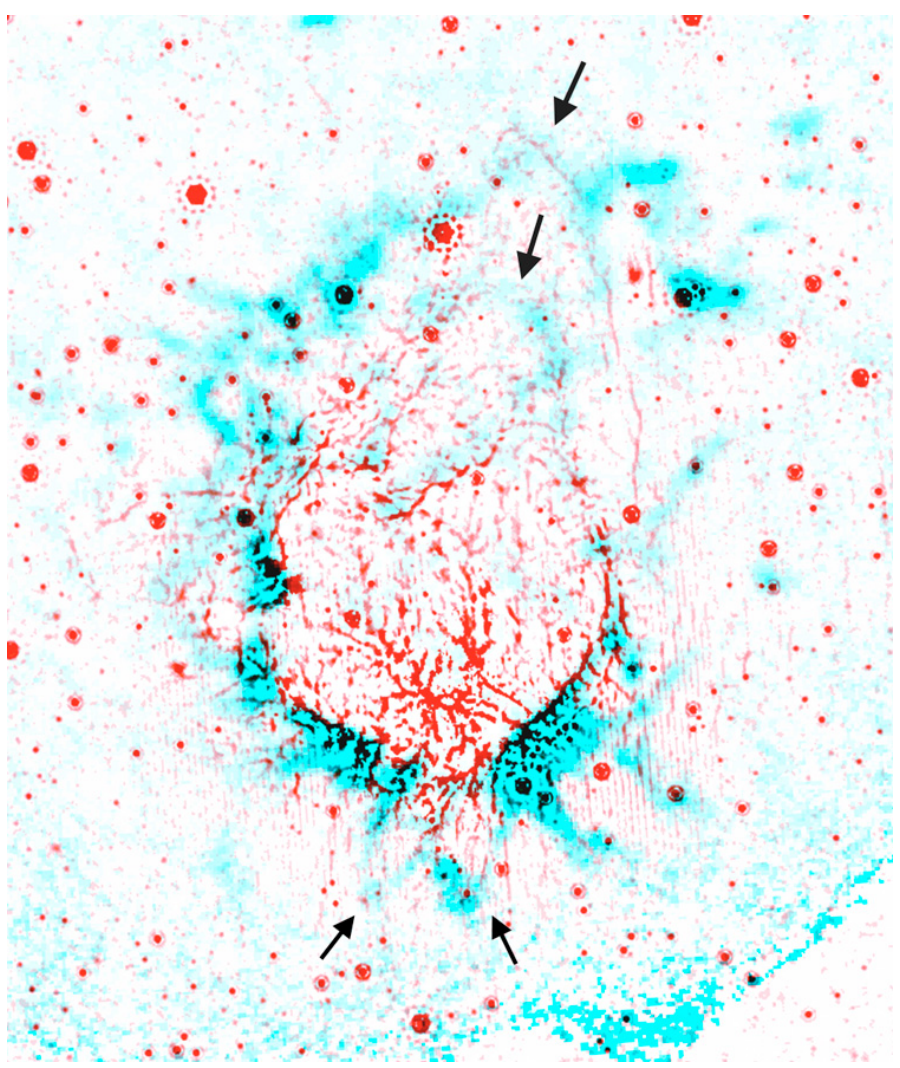

Fig. 16. Colour composite image, with the $870 \mu \mathrm{m}$ emission in turquoise, and in red an unsharp-masked image at $24 \mu \mathrm{m}$ which emphasizes the small-scale low intensity structures of the dust emission from both the ionized region and the surrounding PDR. The black arrows at the top of the figure point to the collected neutral material closing the northern extension of the ionized bubble. The arrows at the bottom of the figure point to the bubbles formed by the ionized gas flowing away from the central $\mathrm{H}$ II region.

$\mathrm{H} \alpha$ emission of the ionized gas inside the bubble, and in pink a zone of diffuse low intensity $\mathrm{H} \alpha$ emission from the PDR surrounding the bubble. This ionized PDR is extended. The unsharp-masked image at $24 \mu \mathrm{m}$ shows the structures of the dust emission from the PDR, covering about the same zone as the diffuse $\mathrm{H} \alpha$ emission. The ionized PDR extends over an area with about twice the radius of the central H II region. As stressed by $\mathrm{ZA} 07$, the IF is porous, and the ionizing radiation escapes to the outside through small holes. This ionized PDR is probably of low density, but its existence demonstrates the long-distance influence of the $\mathrm{H}$ II region and its central exciting star.

The $870 \mu \mathrm{m}$ emission map shows radial features inside condensation 5 and also in the southern parts of RCW 120; there two bubbles are observed between condensations 1 and 2 (see the black arrows at the bottom of Fig. 16). Fig. 4 shows, at $8.0 \mu \mathrm{m}$ and with a high resolution, the radial structures inside condensation 5; only the pressure of the ionized gas seems able to form filaments perpendicular to the IF.

Thus star formation can probably be triggered far from the ionization front - for example in condensation 6 , which is in contact with the extended PDR.

\subsection{An unanswered question}

A question raised by ZA07 remains unanswered: what is the three-dimensional geometry of the RCW 120 bubble? Is there

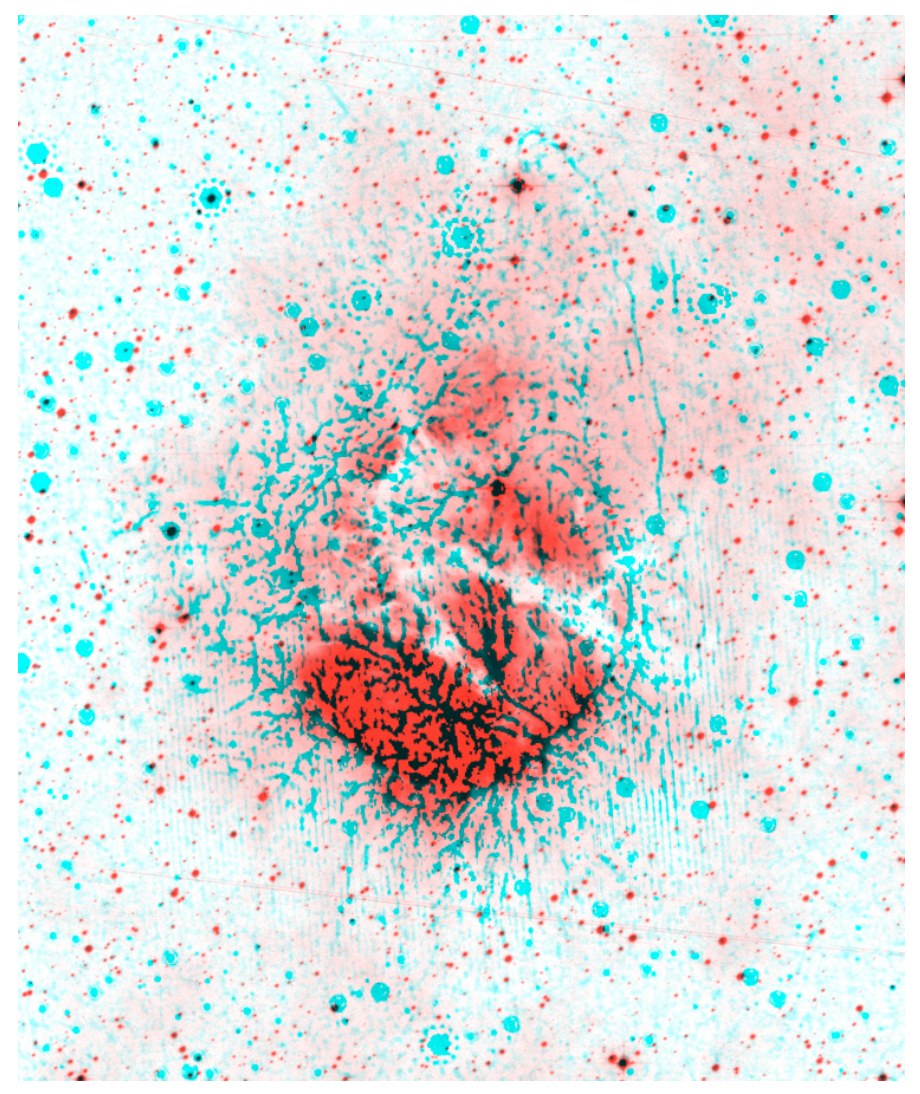

Fig. 17. Colour composite image, with the $\mathrm{H} \alpha$ emission of the ionized gas in red, and the unsharp-masked image at $24 \mu \mathrm{m}$ in turquoise.

a preferential plane for the accumulation of neutral material during the expansion of RCW 120? We have identified only two Class I sources in the direction of the ionized gas (\#61 and \#65), whereas more should be observed if star formation is at work in a spherical collected shell. According to the simulations of Krumholtz et al. (2007), the presence of a magnetic field inhibits motion of the gas across magnetic field lines, leading to a concentration of collected material in a preferential plane. This point, important for the study of star formation triggered by H II regions, will possibly be addressed via submillimetre polarimetric observations.

\section{Conclusions}

In this paper we present new observations, obtained with the APEX-LABOCA camera, of the thermal dust emission at $870 \mu \mathrm{m}$. The higher angular resolution and signal-to-noise ratio of these observations, compared to the previous $1.2-\mathrm{mm}$ observations, allow the detection of low brightness structures and permit more accurate mass estimates. The observations confirm the presence of a shell of dense neutral material collected around the ionized region during its expansion. This shell is now observed all around RCW 120, even at the border of its northern extension. The shell's mass is greater than $2000 M_{\odot}$; we confirm that it is fragmented, with massive fragments of a few tens or hundreds of solar masses. We also confirm that a very massive $\left(\sim 250 M_{\odot}\right)$ and compact $(0.095 \mathrm{pc} \times 0.05 \mathrm{pc})$ core lies adjacent to the ionization front in the most massive fragment.

We present and discuss mid-IR observations at $24 \mu \mathrm{m}$ and $70 \mu \mathrm{m}$, obtained by Spitzer-MIPSGAL. We have measured 138 sources at $24 \mu \mathrm{m}$ and six at $70 \mu \mathrm{m}$. These observations provide new indicators to better distinguish between stars and YSOs, and 
to better distinguish between the evolutionary stages (Class I or Class II) of the sources. Robitaille et al. (2006) have shown the uncertainties tainting these classifications. RCW 120 is a good illustration of the difficulty of this study. The main uncertainty is due to the objects' external extinction, which is not known; highly reddened stars can be mistaken for Class II sources, and highly reddened Class II sources for Class I sources. We have used the $870 \mu \mathrm{m}$ emission to determine the maximum external extinction which may affect a given $24 \mu \mathrm{m}$ source.

We have detected 21 Class I sources, 18 sources possibly intermediate between Class I and Class II, 29 Class II sources, and nine more uncertain Class II-Class III sources. A large fraction of the Class I or intermediate Class I-Class II sources are observed in the direction of the shell of collected neutral material surrounding the ionized region. This distribution demonstrates that their formation has been triggered by the expanding H II region.

As stressed by ZA07, we believe that several different mechanisms are at work simultaneously to form stars in the collected layer. The mechanisms that we tentatively identify are smallscale gravitational instabilities in condensation 1, dynamical instabilities of the IF in condensation 2, and interaction of the IF with a pre-existing molecular condensation in condensation 4 . The collect \& collapse process, which implies large-scale gravitational instabilities of the collected layer along its surface, is possibly at work in the dense core of condensation 1 , which harbours a source detected only at $70 \mu \mathrm{m}$. High-resolution kinematic observations are necessary to ensure that a Class 0 source is present there, building its mass by accreting material in the dense core; enough mass is present there $\left(\sim 250 M_{\odot}\right)$ to allow the formation of a massive star.

We have also detected, at $24 \mu \mathrm{m}$, in the direction of condensation 1, a very unusual and remarkable feature: a chain of about eleven Class I or flat spectrum sources, parallel to the IF. These YSOs have approximately solar masses, and are regularly spaced by some $0.1 \mathrm{pc}$. To our knowledge it is one of the best illustrations ever found, in the domain of star formation, of the Jeans gravitational instability at work.

Finally, we have shown that the HII region, via the UV radiation of its central exciting star, has a long distance influence upon its surrounding medium. If $R$ is the radius of the $\mathrm{H}$ II region, the low density ionized PDR, and the associated dust emitting at $24 \mu \mathrm{m}$, extends a further $R$ outward from the IF. This influence is also reflected by the shape of the collected layer, which shows radial features and bubbles. We conclude that the $\mathrm{H}$ II region can probably trigger star formation far from the IF. This remains to be confirmed by other examples of $\mathrm{H}$ II regions.

Acknowledgements. We thank the APEX staff for their support during the observations with LABOCA, and A. Beelen who performed the first reduction of the data. We thank the anonymous referee for his suggestions and comments. This research has made use of the SIMBAD data base, operated at CDS, Strasbourg, France, and of the interactive sky atlas Aladin (Bonnarel et al. 2000). This work is based in part on observations made with the Spitzer Space Telescope, which is operated by the Jet Propulsion Laboratory, California Institute of Technology, under contract with NASA. We have made use of the NASA/IPAC Infrared Science Archive to obtain data products from the 2MASS, Spitzer-GLIMPSE and Spitzer-MIPS surveys.

\section{References}

Allen, L. E., Calvet, N., D’Alessio, P., et al. 2004, ApJS, 154, 363 Avedisova, V. S., \& Kondratenko, G. I. 1984, Nauchnye Informatsii, 56, 59 Benjamin, R. A., Churchwell, E., Babler, B. L., et al. 2003, PASP, 115, 953 Bohlin, R. C., Savage, B. D., \& Drake, J. F. 1978, ApJ, 224, 132

Bonnarel, F., Fernique, P., Bienayme, O., et al. 2000, A\&ASS, 143, 33

Carey, S. J., Noriega-Crespo, A., Price, S. D., et al. 2005, BAAS, 37, 1252 Churchwell, E., Povich, M. S., Allen, D., et al. 2006, ApJ, 649, 759

Deharveng, L., \& Zavagno, A. 2008, Handbook of Star Forming Regions, Vol. II, ed. B. Reipurth

García-Segura, G., \& Franco, J. 1996, ApJ, 469, 171

Henning, Th., Michel, B., \& Stognienko, R. 1995, P\&SS, 43, 1333

Hildebrand, R. H. 1983, Q. Jl. R. astr. Soc., 24, 267

Houk, N. 1982, Michigan Catalogue of Two-dimensional Spectral Types for the HD stars (Ann Arbor: Univ. Michigan), 3

Indebetouw, R., Mathis, J. S., Babler, B. L., et al. 2005, ApJ, 619, 931

Indebetouw, R., Robitaille, T. P., Whitney, B. A., et al. 2007, ApJ, 666, 321

Johnstone, D., Matthews, H., \& Mitchell, G. F. 2006, ApJ, 639, 259

Kessel-Deynet, O., \& Burkert, A. 2003, MNRAS, 338, 545

Kirk, H., Johnstone, D., \& Di Franchesco, J. 2006, ApJ, 646, 1009

Krumholz, M. R., Stone, J. M., \& Gardiner, T. A. 2007, ApJ, 671, 518

Lada, C. J., \& Adams, F. C. 1992, ApJ, 393, 278

Lefloch, B., \& Lazareff, B. 1994, A\&A, 289, 559

Martins, F., \& Plez, B. 2006, A\&A, 457, 637

Motte, F., Schilke, P., \& Lis, D. C. 2003, ApJ, 582, 277

Ossenkopf, V., \& Henning, T. 1994, A\&A, 291, 943

Parker, Q. A., Phillipps, S., Pierce, M. J., et al. 2005, MNRAS, 362, 689

Rebull, L. M., Stapelfeldt, K. R., Evans II, N. J., et al. 2007, ApJS, 171, 447

Rieke, G. H., \& Lebofsky, M. J. 1985, ApJ, 288, 618

Robitaille, T. P., Whitney, B. A., Indebetouw, R., Wood, K., \& Denzmore, P. 2006, ApJS, 167, 256

Robitaille, T. P., Whitney, B. A., Indebetouw, R., \& Wood, K. 2007, ApJS, 169, 328

Robitaille, T. P., Meade, M. R., Babler, B. L., et al. 2008, AJ, 136, 2413

Siringo, G., Weiss, A., Kreysa, E., et al. 2007, The Messenger, 129, 2

Stetson, P. B. 1987, PASP, 99, 191

Sugitani, K., Tamura, M., Nakajima, Y., et al. 2002, ApJ, 565, L25

Tokunaga, A. T. 2000, Allen's Astrophysical Quantities, 4th edition, ed. A. N. Cox (New York: Springer-Verlag), 143

Vig, S., Testi, L., Walmsley, M., et al. 2007, A\&A, 470, 977

White, G. J., Nelson, R. P., Holland, W. S., et al. 1999, A\&A, 342, 233

Whitney, B. A., Wood, K., Bjorkman, J. E., \& Wolff, M. J. 2003a, ApJ, 591, 1049

Whitney, B. A., Wood, K., Bjorkman, J. E., \& Cohen, M. 2003b, ApJ, 598, 1079

Whitney, B. A., Indebetouw, R., Bjorkman, J., \& Wood, K. 2004, ApJ, 617, 1177

Young, C. H., Shirley, Y. L., \& Evans II, N. J. 2003, ApJS, 145, 111

Zavagno, A., Pomarès, M., Deharveng, L., et al. 2007, A\&A, 472, 835 (ZA07) 